\title{
THE WIDTH-VOLUME INEQUALITY
}

\author{
LARRY GUTH
}

\begin{abstract}
We prove that a bounded open set $U$ in $\mathbb{R}^{n}$ has k-width less than $C(n)$ Volume $(U)^{k / n}$. Using this estimate, we give lower bounds for the kdilation of degree 1 maps between certain domains in $\mathbb{R}^{n}$. In particular, we estimate the smallest (n-1)-dilation of any degree 1 map between two ndimensional rectangles. For any pair of rectangles, our estimate is accurate up to a dimensional constant $C(n)$. We give examples in which the (n-1)-dilation of the linear map is bigger than the optimal value by an arbitrarily large factor.
\end{abstract}

This paper proves some estimates having to do with the areas of $\mathrm{k}$-dimensional surfaces in Euclidean space. We deal with two problems. First, suppose that $U$ is a bounded open set in $\mathbb{R}^{n}$. We consider the problem of sweeping out $U$ with $\mathrm{k}$-dimensional surfaces, trying to arrange that the volumes of all the surfaces are as small as possible. Depending on the geometry of $U$, we give upper and lower bounds for the possible volumes of the surfaces. In particular, we construct a family of k-dimensional surfaces sweeping out $U$ so that each surface has volume bounded by $C(n)$ Volume $(U)^{k / n}$. The next question concerns mappings from one open set to another - for example from the unit cube to a long thin cylinder. After we fix a domain and a range, we consider the problem of finding a degree 1 mapping which stretches the volumes of k-dimensional surfaces as little as possible. For certain pairs of (n-dimensional) rectangles, we show that the linear mapping stretches the $\mathrm{k}$-dimensional surfaces far more than necessary. We give upper and lower bounds for the minimal amount of stretching by any degree 1 map. When $k=n-1$, these upper and lower bounds match up to a constant factor.

The definition of k-width

As a first approximation to the definition of width, we define a linear version of $\mathrm{k}$-dimensional width. Let $U$ be a bounded open set in $\mathbb{R}^{n}$. For each (n-k)-plane $P$ through the origin, let $F(P)$ denote the family of all k-planes perpendicular to $P$. Define the width of $F(P)$ to be the maximum volume of the intersection of $U$ with any of the k-planes in $F(P)$. Then define the linear k-width of $U$ to be the minimum width of $F(P)$ as $P$ varies among all the (n-k)-planes through the origin.

The width considered in this paper is a non-linear generalization of the definition above. Instead of families of parallel $\mathrm{k}$-planes, we consider families of $\mathrm{k}$-dimensional surfaces. The surfaces we consider will be oriented relative k-cycles in $U$. For the reader not familiar with $\mathrm{k}$-cycles, there is no harm in picturing $\mathrm{k}$-dimensional submanifolds of $U$ with boundary in $\partial U$. We define the k-width of a family $F$ to be the largest $\mathrm{k}$-volume of any of the k-cycles in $F$. In order to define the $\mathrm{k}$ width of $U$, we look at families of cycles that "sweep out" $U$. Morally, a closed (n-k)-dimensional family $F$ of k-cycles can be glued together to form a single ndimensional cycle. If this n-dimensional cycle has a non-zero homology class, then we say that $F$ "sweeps out" $U$. For example, if $\pi$ is a generic PL map from $U$ to 
$\mathbb{R}^{n-k}$, then the fibers $\pi^{-1}(y)$ form a family of k-cycles sweeping out $U$, parametrized by $y \in \mathbb{R}^{n-k}$. We define the k-width of $U$ to be the smallest k-width of any family of k-cycles sweeping out $U$. Because the definition doesn't involve planes, it also makes sense if we replace $U$ by any compact oriented Riemannian manifold.

Mathematicians working on geometric measure theory began to look at families of cycles in the 1960's. In the unpublished paper [1], Almgren used such families as a tool to construct minimal cycles on a Riemannian manifold using Morse-theoretic arguments. A good reference for this material is the first chapter of Pitts's book 12. Gromov had the idea to use families of cycles as a way of describing the size of a Riemannian manifold $(M, g)$. He sketched his ideas about this subject in section $\mathrm{F}$ of appendix 1 of his long paper on metric geometry [7. In this section he essentially gave the definition above.

Because the space of all k-cycles is infinite dimensional, it takes some work to prove that the k-width of an open set is not zero. The first proof of this fact is essentially due to Almgren. Gromov pointed out that Almgren's work establishes the exact $\mathrm{k}$-width of the unit $\mathrm{n}$-sphere: the $\mathrm{k}$-width of the unit $\mathrm{n}$-sphere is equal to the volume of the unit k-sphere. Almgren's proof requires a substantial amount of geometric measure theory. Gromov also gave an elementary lower bound for the $\mathrm{k}$-width of the unit n-sphere. Using Gromov's proof, it's not hard to estimate the $\mathrm{k}$-width of simple shapes like rectangles.

Proposition 1. Let $R$ be an $n$-dimensional rectangle with dimensions $R_{1} \leq \ldots \leq$ $R_{n}$. Then the $k$-width of $R$ is at least $c(n) R_{1} \ldots R_{k}$ and at most $R_{1} \ldots R_{k}$.

(It seems reasonable to guess that the k-width of a rectangle is $R_{1} \ldots R_{k}$, but the exact value of the $\mathrm{k}$-width is unknown.)

The width-volume inequality

The first theorem of this paper is an upper bound for the k-width of sets with small volume in Euclidean space.

Theorem 1. (The width-volume inequality) Let $U$ be a bounded open set in $\mathbb{R}^{n}$ with volume $V(U)$ and $k$-width $W_{k}(U)$. Then $W_{k}(U)<C(n) V(U)^{k / n}$.

To prove the theorem, we have to construct a family of cycles that sweep out $U$ in an efficient way. The first approach one might try is to use parallel planes at a wellchosen angle, as in the definition of linear k-width. This approach can fail because of the Kakeya phenomenon. As proven by Besicovitch, there are open sets $U$ in $\mathbb{R}^{2}$ with arbitrarily small area containing a unit line segment in every direction. These sets have linear 1-width at least 1 and arbitrarily small area. A good reference for Besicovitch sets is Wolff's article [15]. While I was revising this paper, I learned that taking parallel k-planes does work when $k>n / 2$. This result was proven by Falconer in the interesting short paper [5] (in slightly different language). In the last section, we briefly explain Falconer's proof, which is based on Fourier analysis. For the intermediate range $2 \leq k \leq n / 2$, I don't know if the linear k-width of a set $U$ can be bounded in terms of its volume. There are some more comments in the open problem section.

We now sketch the proof of our theorem, which deals with all values of $\mathrm{k}$. Because of a scaling argument, we can assume that the volume of $U$ is 1 . The first step of the argument is to find a lot of k-planes that meet $U$ in a small volume. We find these planes by an averaging trick. Let $S_{0}$ be the k-skeleton of the unit lattice in 
$\mathbb{R}^{n}$. We consider the translations of $S_{0}$ by a vector $x \in[0,1]^{n}$. On average, the translation of $S_{0}$ meets $U$ in a region of volume $\left(\begin{array}{l}n \\ k\end{array}\right)$. Therefore, we can choose a translate $S$ of $S_{0}$ which meets $U$ in a controlled volume. We can then control the k-volume of any k-cycle lying in the skeleton $S$.

It is not possible to sweep out $U$ with cycles lying in the skeleton $S$, because any family of cycles sweeping out $U$ must pass through every point of $U$. But it turns out to be possible to sweep out $U$ by a family of k-cycles each of which lies in $S$ except for a subset of controlled volume.

Proposition 2. For any bounded open set $V \in \mathbb{R}^{n}$ (of any volume), there is a family of $k$-cycles sweeping out $V$ so that each cycle lies in $S$, except for a subset of volume at most $C(n)$.

We include some pictures to indicate how such a family might look for $k=1$, $n=2$. The thin lines denote the 1-skeleton $S$ and the thick lines denote a 1-cycle in our family.

1.

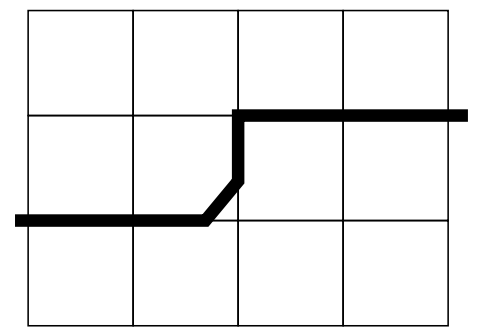

3.

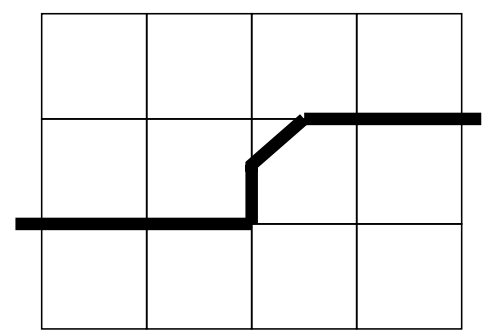

2.

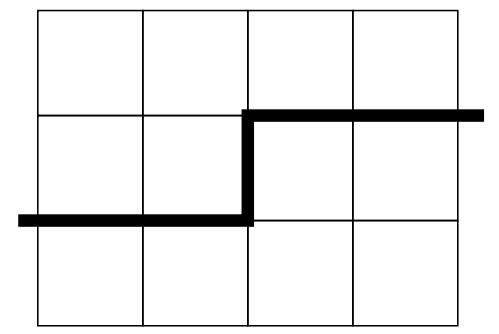

4.

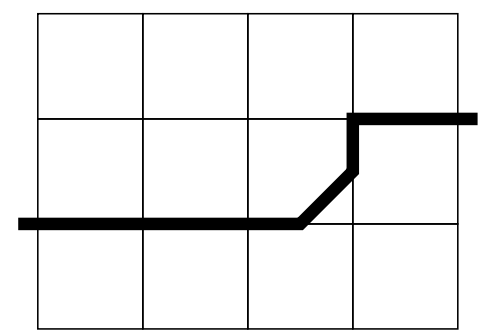

Figure 1

The general case is somewhat harder than the case $k=1, n=2$. In general, the family of k-cycles is constructed by starting with a family of parallel k-planes transverse to $S$, and then "bending" them so that almost all of the volume of each k-plane is pushed into the skeleton $S$. We call this construction "bending planes around a skeleton".

\section{Area-contracting maps between rectangles}

In the second half of the paper, we apply the width-volume inequality to estimate the k-dilations of degree 1 maps. Recall that k-dilation measures how much a mapping stretches $\mathrm{k}$-dimensional volumes. If a map $f$ takes any $\mathrm{k}$-dimensional manifold with volume $V$ to an image with volume at most $\lambda V$, then $f$ has k-dilation at most $\lambda$. 
The second main problem of this paper is to estimate the infimal k-dilation of all degree 1 maps from a rectangle $R$ to another rectangle $S$. This innocuous-sounding problem has turned out to be much more complicated than I expected. When I first approached the problem, I guessed that a linear diffeomorphism from $R$ to $S$ would give at least roughly the smallest $\mathrm{k}$-dilation. My guess was wrong. Let us define $D_{k}(R, S)$ to be the infimal k-dilation of any degree 1 map from $R$ to $S$ (taking the boundary of $R$ to the boundary of $S)$. For comparison, let us define $\operatorname{Lin}_{k}(R, S)$ to be the smallest k-dilation of a linear diffeomorphism from $R$ to $S$.

Proposition 3. For each $n \geq 3$ and each $k$ in the range $2 \leq k \leq n-1$, there are pairs of $n$-dimensional rectangles $(R, S)$ so that the ratio $\operatorname{Lin}_{k}(R, S) / D_{k}(R, S)$ is arbitrarily large.

For example, if the rectangle $R$ has dimensions $\epsilon \times 1 \times 1$, and the rectangle $S$ has dimensions $\epsilon \times \epsilon \times \epsilon^{-1}$, then $\operatorname{Lin}_{2}(R, S)=\epsilon^{-1}$. On the other hand, there is a non-linear degree 1 map from $R$ to $S$ with 2-dilation less than 1000, regardless of $\epsilon$. I call this map the snake map because it somewhat resembles a snake uncoiling.

We take a little time to describe this map. The snake map does not have any analogue in 2 dimensions, but there is a map related to it. Let $U$ be the unit square, and let $A \subset U$ be the shape in Figure 2 .

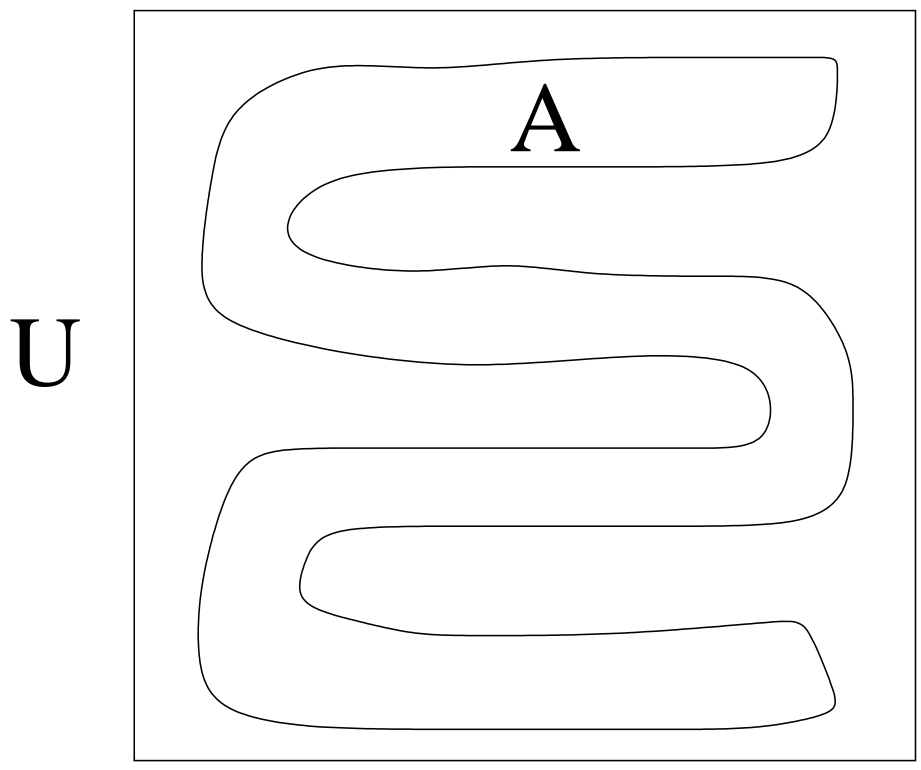

Figure 2

The set $A$ is bilipschitz to the rectangle $[0, \epsilon] \times\left[0, \epsilon^{-1}\right]$, and it snakes back and forth across $U$ roughly $\epsilon^{-1}$ times. Let $A^{c}$ denote the complement of $A$ in $U$. The first map that we consider is a retraction $\phi$ of $U$ onto $A$, which maps $A^{c}$ onto $\partial A$. The 1-dilation of $\phi$ is roughly $\epsilon^{-1}$ and the 2 -dilation of $\phi$ is exactly 1 .

We now turn to three dimensions. The rectangle $R$ is equal to $[0, \epsilon] \times U$ and the rectangle $S$ is bilipschitz to $[0, \epsilon] \times A$. We can get a degree 1 map from $R$ to $S$ by first retracting $R$ onto $[0, \epsilon] \times A$ and then using the bilipschitz equivalence of $[0, \epsilon] \times A$ with $S$. 
The most obvious retraction from $R$ onto $[0, \epsilon] \times A$ is $i d \times \phi$, where $i d$ denotes the identity map from $[0, \epsilon]$ to itself. This retraction has 2-dilation roughly $\epsilon^{-1}$. Using this retraction, we get a degree 1 map from $R$ to $S$ with 2-dilation roughly $\epsilon^{-1}$, slightly larger than the 2-dilation of the linear map.

The trick in the construction of the snake map is to improve the retraction from $R$ to $[0, \epsilon] \times A$. The improved retraction takes place in two steps. We first retract $R$ onto the union $(\{0\} \times U) \cup([0, \epsilon] \times A)$. We then retract this set onto $[0, \epsilon] \times A$. The set $(\{0\} \times U) \cup([0, \epsilon] \times A)$ resembles a snake sitting on a piece of cardboard. The first retraction can be done with 1-dilation roughly 1, and hence 2-dilation roughly 1 also. The second retraction is accomplished by the map $i d \times \phi$. The second retraction has 1 -dilation roughly $\epsilon^{-1}$ but it has 2 -dilation 1 . To check the 2 dilation of the retraction, we reason as follows. The restriction of $i d \times \phi$ to $[0, \epsilon] \times A$ is the identity, and so it has 2-dilation 1 . But the complement of $[0, \epsilon] \times A$ in the domain of our map is just $\{0\} \times A^{c}$. Our retraction maps this 2 -dimensional set to the 1 -dimensional set $\{0\} \times \partial A$. Therefore, the second retraction has 2-dilation 1 .

\section{Lower bounds for the k-dilation}

Next we approach the problem from the other side, proving lower bounds for the k-dilation $D_{k}(R, S)$. Our lower bounds are based on k-width and on the widthvolume inequality. Our estimates for $D_{k}(R, S)$ depend on the dimensions of $R$ and $S$. We adopt the convention that $R$ and $S$ are n-dimensional rectangles, that $R$ has dimensions $R_{1} \leq \ldots \leq R_{n}$ and that $S$ has dimensions $S_{1} \leq \ldots \leq S_{n}$.

The first lower bound on $D_{k}(R, S)$ comes from knowing the k-width of $S$. Suppose that $f$ is a degree 1 map from $R$ to $S$ with k-diliation $\lambda$. The rectangle $R$ can be sliced into k-dimensional rectangles with dimensions $R_{1} \times \ldots \times R_{k}$, and these rectangles form a family of cycles sweeping out $R$. The image of each $\mathrm{k}$-dimensional rectangle has volume at most $\lambda R_{1} \ldots R_{k}$. The situation is illustrated in Figure 3.
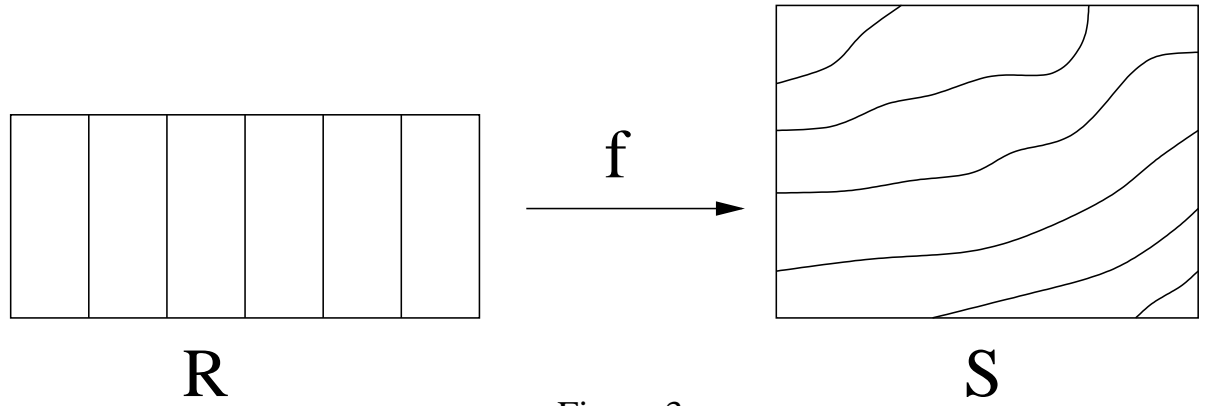

Figure 3

The image of our family of rectangles is a family of k-cycles sweeping out $S$. According to Proposition 1, this family must contain a cycle with volume at least $c(n) S_{1} \ldots S_{k}$. Since each cycle in the family has volume at most $\lambda R_{1} \ldots R_{k}$, we get a lower bound for the k-dilation $\lambda$.

Proposition 4. $D_{k}(R, S)>c(n)\left[S_{1} \ldots S_{k}\right] /\left[R_{1} \ldots R_{k}\right]$.

We can get more estimates if, instead of considering the k-width of $S$, we work with the k-width of subsets of $S$. Let's see how this idea works out in a particular case. Suppose that $S$ is a 3-dimensional rectangle with dimensions $S_{1} \leq S_{2} \leq S_{3}$. Then $S$ contains many subrectangles with dimensions $S_{1} \times S_{2} \times S_{2}$. We can find $N$ disjoint rectangles in $S$ with those dimensions, where $N$ is roughly $S_{3} / S_{2}$. Call the rectangles $V_{i}$. Each one of these rectangles has 2-width roughly $S_{1} S_{2}$. Now 
suppose that $f$ is a degree 1 map from $R$ to $S$ with 2-dilation $\lambda$. Then each of our rectangles has a preimage $U_{i}=f^{-1}\left(V_{i}\right)$, and each of these preimages has 2-width at least $S_{1} S_{2} / \lambda$. The situation is illustrated in Figure 4 .
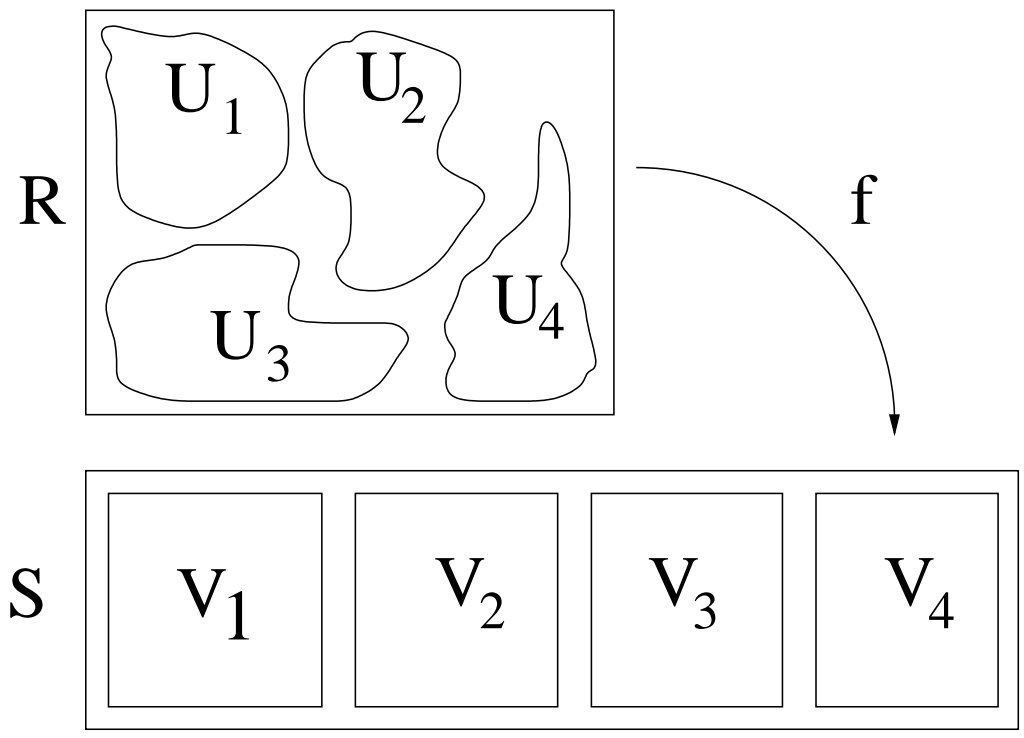

Figure 4

We want to use this information to get a lower bound on $\lambda$. Since the sets $U_{i}$ are disjoint, one of them must have volume at most $R_{1} R_{2} R_{3} / N$. We are led to the following question: if $U \subset R$ is an open set with volume $V(U)$, what is the largest possible 2-width of $U$ ? Since $U$ is a subset of $R$, its 2 -width is at most $R_{1} R_{2}$. For large volumes $V(U)$, this upper bound is the best possible, but for smaller volumes it can be improved. Using the width-volume inequality, we can bound the 2-width of $U$ by $C V(U)^{2 / 3}$. This upper bound is sharp for small volumes $V(U)$. These upper bounds can be improved if $V(U)$ is in the intermediate range $R_{1}^{3}<<V(U)<<R_{1} R_{2}^{2}$. An example of a set $U$ with 2-width roughly $V(U)^{2 / 3}$ is the round ball of volume $V(U)$, which has radius roughly $V(U)^{1 / 3}$. If $R_{1}^{3}<<V(U)$, then this round ball does not fit in the rectangle $R$. It turns out that all subsets of $R$ with volume $V(U)$ are substantially thinner than the round ball. We make this precise in the following proposition.

Proposition 5. Let $R$ be a 3-dimensional rectangle with dimensions $R_{1} \leq R_{2} \leq$ $R_{3}$. Suppose that $U \subset R$ is an open set with volume $V(U)$. Then the 2-width of $U$ is at most $C R_{1}^{1 / 2} V(U)^{1 / 2}$.

This estimate is a variation on the width-volume inequality adapted to subsets of the rectangle $R$. It improves on the original inequality exactly when $R_{1}^{3}<<V(U)$. The proof is only a small modification of the proof of the width-volume inequality. Using this inequality to upper bound the 2-width of one of the sets $U_{i}$, we get a new lower bound for $D_{2}(R, S)$.

Proposition 6. If $R$ and $S$ are 3-dimensional rectangles with dimensions $R_{1} \leq$ $R_{2} \leq R_{3}$ and $S_{1} \leq S_{2} \leq S_{3}$, then $D_{2}(R, S)>c\left[S_{1} S_{2}^{1 / 2} S_{3}^{1 / 2}\right] /\left[R_{1} R_{2}^{1 / 2} R_{3}^{1 / 2}\right]$. 
In the paper we carry out this idea for all values of $\mathrm{k}$ and $\mathrm{n}$, proving lower bounds for $D_{k}(R, S)$. In the special case that $k=n-1$, our lower bounds and the maps we will construct match up well enough to determine $D_{n-1}(R, S)$ up to a constant factor.

Theorem 2. Let $R$ and $S$ be $n$-dimensional rectangles. Let $R$ have dimensions $R_{1} \leq \ldots \leq R_{n}$, and $S$ have dimensions $S_{1} \leq \ldots \leq S_{n}$. Let $Q_{i}$ denote the quotient $S_{i} / R_{i}$. Up to a constant factor $C(n)$, the optimal $(n-1)$-dilation $D_{n-1}(R, S)$ is equal to the maximum of the following list of $n$ monomials in the variables $Q_{i}$.

The first $n-1$ monomials are given by $Q_{1} \ldots Q_{l}\left(Q_{l+1} \ldots Q_{n}\right)^{\frac{n-l-1}{n-l}}$, where $l$ is an integer in the range $1 \leq l \leq n-1$. The final monomial is $Q_{2} \ldots Q_{n}$.

The algebra here is somewhat complicated, but the complicated expressions in $Q_{i}$ are not the important point. We have seen that the snake map can have (n-1)dilation much smaller than that of the linear map. For any two rectangles $R$ and $S$ we will construct an explicit map with nearly optimal (n-1)-dilation. Depending on the rectangles, it may be a linear map, or it may be a minor generalization of the snake map. Up to a constant factor, the expression in the theorem will turn out to be the (n-1)-dilation of this map. The lower bounds in the theorem guarantee that the (n-1)-dilation of this map cannot be substantially improved. (On the other hand, for $2 \leq k<n-1$, the k-dilation of the snake map can be improved in some cases. For more information on this problem, see [9].)

\section{Related results and open questions}

The literature contains a couple of theorems in a similar spirit to the widthvolume inequality. For example, in appendix 1 of 7 , page 128, Gromov proved the following estimate connecting the Uryson width and the area of a Riemannian 2-sphere. (The Uryson width is a different notion of width from the one in this paper. For a definition, see Gromov's book [8], page 108.)

Theorem. (Gromov) Let $\left(S^{2}, g\right)$ be a Riemannian 2-sphere with Uryson 1-width $W$ and area $A$. Then $W<2 A^{1 / 2}$.

Another geometric quantity related to the k-width is the volume of the smallest stationary k-cycle in a Riemannian manifold. According to the work of Almgren [1, a closed oriented Riemannian manifold $(M, g)$ contains a stationary k-dimensional varifold with volume at most $W_{k}(M, g)$. Recently, Nabutovsky and Rotman proved several estimates for the length of the shortest stationary 1-cycle in a Riemannian manifold. One important estimate is the following theorem from [1].

Theorem. (Nabutovsky, Rotman) A closed Riemannian n-manifold $(M, g)$ of volume $V$ contains a stationary 1-cycle of length at most $C(n) V^{1 / n}$.

Although these theorems are in a similar spirit to Theorem 1, they don't give any upper bounds for $\mathrm{k}$-width for any value of $\mathrm{k}$. These theorems hold in a more general setting than Theorem 1 because they apply to arbitrary Riemannian metrics, whereas Theorem 1 applies only to domains in Euclidean space.

Comparing our result to the results of Gromov, Rotman, and Nabutovsky, it seems reasonable to ask whether there is a width-volume inequality for all Riemannian manifolds. We phrase this as a problem.

Open Problem. For which integers $k<n$ is there a constant $C(k, n)$ so that for every closed oriented Riemannian n-manifold $(M, g)$, the $k$-width is bounded in terms of the volume by the formula $W_{k}(M, g)<C(k, n) \operatorname{Volume}(M, g)^{k / n}$ ? 
In an appendix to this paper, we show that the answer to this question is negative when $k=n-1$. In other words, a closed oriented Riemannian n-manifold may have volume 1 and arbitrarily large (n-1)-width. For $k<n-1$, the problem is open.

Now we turn to some other open problems related to k-width.

Open Problem. If $k$ is in the range $2 \leq k \leq n / 2$, is it possible to bound the linear $k$-width of a bounded open set $U \subset \mathbb{R}^{n}$ in terms of its volume?

This problem is related to the problem of Besicovitch $(n, k)$ sets. An $(n, k)$ set is defined to be a subset of $\mathbb{R}^{n}$ of Lebesgue measure zero containing a translate of every k-plane. Besicovitch gave examples of $(2,1)$ sets, and an easy generalization shows that $(n, 1)$ sets exist for all $\mathrm{n}$. On the other hand, Falconer's theorem mentioned above proves that there are no $(n, k)$ sets for $k>n / 2$. There is recent progress on this problem starting with Bourgain's important paper [2] on the Kakeya maximal function. Bourgain proves that there are no $(4,2)$ sets and no $(7,3)$ sets, among other results. The problem of $(n, k)$ sets, however, is not equivalent to the problem above. For example, to prove that there are no $(4,2)$ sets, Bourgain establishes the following slightly weaker version of a linear width-volume inequality. (The theorem below follows immediately from Proposition 3.3 in 2.)

Theorem. (Bourgain) For each $\epsilon>0$, there is a constant $C_{\epsilon}$ so that the following estimate holds. Let $U$ be a bounded open subset in $\mathbb{R}^{4}$, with volume $V(U)$ and diameter $D(U)$. Then the linear 2-width of $U$ is bounded by $C_{\epsilon} V(U)^{\frac{2-\epsilon}{4}} D(U)^{\epsilon}$.

Open Problem. Find the $k$-width of the unit n-cube.

The exact k-width of the unit n-sphere was determined by Almgren, and in [7, Gromov claims that the $\mathrm{k}$-width of the unit $\mathrm{n}$-ball is exactly the volume of the unit $\mathrm{k}$-ball. Because a cube contains a ball, the results of Almgren and Gromov show that the k-width of the unit $\mathrm{n}$-cube is bounded below by a constant independent of $n$. The linear k-width of the unit n-cube is known to be 1 by a result of Vaaler 13. Even this result is difficult. Another interesting problem along these lines, described by Gromov in [7, is to estimate the k-width of the unit ball in the finitedimensional Banach space $l^{\infty}(n)$. Gromov showed that an estimate independent of $\mathrm{n}$ would lead to results in intrinsic Riemannian geometry.

Open Problem. (The sponge problem) Recall that an embedding I is called expanding if its derivative increases the length of all vectors, or equivalently if it increases the lengths of all curves. For which dimensions $n$ is there a constant $\epsilon>0$ so that any bounded open set $U \subset \mathbb{R}^{n}$ with volume less than $\epsilon$ admits an expanding embedding into the unit $n$-ball?

A potential counterexample $U$ must have a small volume and a large diameter. It might resemble a sponge: a large cube from which many tubes have been cut leaving a complicated region with small volume. The expanding embedding reminds me of squeezing the water out of the sponge. An affirmative answer to the sponge problem would give a new proof of the width-volume inequality, because the image of $U$ can be swept out by k-planes with volume 1, and the inverse images of these k-planes sweep out $U$ with bounded volume. I tried for a long time to construct the expanding embeddings, but I wasn't able to do it even in dimension 2.

Open Problem. Estimate $D_{k}(R, S)$ up to a constant factor, for $k$ in the range $2 \leq k \leq n-2$. 
The cases $k=1$ and $k=n$ are elementary, and the case $k=n-1$ is done in this paper. This paper contains some lower bounds for $D_{k}(R, S)$. It also includes some degree 1 mappings which give upper bounds for $D_{k}(R, S)$. There are several more upper and lower bounds for $D_{k}(R, S)$ in 9 . The gap between the best upper and lower bounds can be arbitrarily large, however. The work in [9] suggests that the cases $2 \leq k \leq n-2$ are a lot harder than the case $k=n-1$. This specific problem may not have any applications, but I think it's a good testing ground to see how well we understand k-dilation.

\section{The plan of the paper}

In the first section of the paper, we give the precise definition of k-width and its basic properties. We give Gromov's proof that the k-width is not zero and estimate the k-widths of cubes and rectangles. In the second section of the paper, we carry out the "bending planes around a skeleton" construction and use it to prove the width-volume inequality. The second section also includes the variation of the width volume inequality adapted to subsets of a rectangle. In the third section, we use these estimates to prove lower bounds for the k-dilation of degree 1 maps. In particular, we prove all the lower bounds in Theorem 2. In the fourth section, we construct the snake map and its higher-dimensional analogues. Using these maps, we check that non-linear maps can have much smaller k-dilation than linear maps. Then we prove all the upper bounds in Theorem 2. The fifth section is an appendix which gives lower bounds for the width of Riemannian manifolds using isoperimetric inequalities. It shows that a Riemannian manifold of volume 1 may have arbitrarily large (n-1)-width. The sixth section is a second appendix, briefly explaining Falconer's bound on the linear k-width for $k>n / 2$.

Throughout the paper we use $c(n)$ and $C(n)$ to denote constants that depend only on the dimension $\mathrm{n}$. The value of these constants may change from line to line. We use $C(n)$ to denote a large constant and $c(n)$ to denote a small positive constant. As described above, when we talk about a rectangle $R$, we always order its dimensions so that $R_{1} \leq \ldots \leq R_{n}$.

This paper is based on a section of my thesis [9], and I would like to thank my advisor Tom Mrowka for his help and support. I am also grateful to the referees for their constructive comments.

\section{The DEFINITION OF K-WIDTH}

In this section, we will make precise the intuitive idea of k-width described in the introduction. Our first task is to give a precise meaning to a family of $\mathrm{k}$ cycles. We will use families of flat integral cycles which are continuous in the flat topology. Roughly speaking, the flat topology means that two k-cycles are close to one another if their difference bounds a $(\mathrm{k}+1)$-chain with small volume. The precise definition that we give is somewhat technical. A reader interested in the main ideas of the paper might skip the definition and proceed with only an intuitive idea of a family of cycles sweeping out M. After defining the k-width, we prove that it behaves monotonically with respect to appropriate mappings and that it is not zero. At the end of the section, we estimate the $\mathrm{k}$-widths of cubes and rectangles.

Our explanation of the flat topology and flat cycles essentially follows Fleming's paper [6]. We recall that an integral Lipschitz k-chain in $(M, g)$ is a finite sum 
$\sum c_{i} f_{i}$, where each $c_{i}$ is an integer and each $f_{i}$ is a Lipschitz map from the ksimplex to M. An integral k-chain is a special case of a singular k-chain with integer coefficients. We define the boundary of a k-chain as in the singular homology theory.

We define the volume of the map $f_{i}$ to be the volume of the k-simplex in the induced metric $f_{i}^{*}(g)$. Then we define the mass of the chain $\sum c_{i} f_{i}$ to be $\sum\left|c_{i}\right|$ volume $\left(f_{i}\right)$. We abbreviate the mass of a k-chain $\mathrm{C}$ by $|C|$. The most important fact about $\mathrm{k}$-chains and mass is the isoperimetric inequality. We will use the following rather weak formulation of the isoperimetric inequality.

Theorem. (Isoperimetric Inequality) For each compact manifold $(M, g)$, there is a constant $\epsilon$ so that every integral Lipschitz $k$-cycle with mass less than $\epsilon$ is homologically trivial. Moreover, if $C$ is a $k$-cycle with mass $m<\epsilon$, then $C=\partial D$ for some integral Lipschitz $(k+1)$-chain $D$ with mass less than $C(n) m$.

Now we define the flat norm. The flat norm was introduced by Whitney in 14. and used to define spaces of cycles by Fleming in [6. The flat norm of a k-chain $\mathrm{C}$ is the infimal value of $|C-\partial D|+|D|$ as $\mathrm{D}$ varies over all the integral Lipschitz $(\mathrm{k}+1)$-chains in M. We define the flat distance between chains $C_{1}$ and $C_{2}$ as the flat norm of $C_{1}-C_{2}$. It may happen that the distance between two flat chains is zero. This occurs when the two chains parametrize the same geometric object in different ways. According to a result of Fleming (see []ㅡ), the distance is zero only for this reason. We do not need this result however. We identify any two chains separated by flat distance zero. The space of equivalence classes is now a metric space. The completion of this metric space is called the space of integral flat k-chains in M.

We define the mass of a flat chain $C$ to be the infimal number $m$ so that there exists a sequence of integral Lipschitz chains $C_{i}$ converging to $C$ with mass less than $m$.

The boundary map on integral Lipschitz chains is bounded with respect to the flat norm. In fact, if $\mathrm{C}$ is a $\mathrm{k}$-chain and $\mathrm{D}$ is a $(\mathrm{k}+1)$-chain, then the flat norm of $\partial C$ is at most $|C-\partial D|$. Taking the infimum over all $\mathrm{D}$ shows that the flat norm of $\mathrm{C}$ is greater than or equal to the flat norm of the boundary of $\mathrm{C}$. Therefore, we can define boundaries of flat chains. The flat k-cycles are the subset of flat k-chains with boundary zero. Let $\mathrm{Z}$ denote the space of flat k-cycles with the flat topology.

By a family $\mathrm{F}$ of k-cycles in $\mathrm{M}$, we mean a continuous map $\mathrm{F}$ from a parameter space $\mathrm{P}$ to $\mathrm{Z}$. We will always assume that $\mathrm{P}$ is a finite simplicial complex. We define the width of the family $\mathrm{F}$ to be $\sup _{p \in P}|F(p)|$.

Our next task is to define what it means for a family of k-cycles to sweep out M. Morally, an i-dimensional family of k-cycles can be glued together to form a $(\mathrm{k}+\mathrm{i})$-cycle, but this is not literally true for the space of flat cycles. We now give a construction that takes an i-dimensional family of cycles and gives a $(\mathrm{k}+\mathrm{i})$-cycle that, in some sense, is a small perturbation of the family.

Let $\mathrm{F}$ be a family of k-cycles parametrized by $\mathrm{P}$. We take a fine triangulation of $\mathrm{P}$. We pick a small number $\delta>0$. For each vertex $\mathrm{v}$ of the triangulation, we choose an integral Lipschitz cycle $C(v)$, with flat distance less than $\delta$ from $F(v)$, and mass less than $|F(v)|+\delta$.

(For completeness, we include the proof that such a cycle $C(v)$ exists. By the definition of a flat cycle, we can take a sequence of integral Lipschitz k-chains $C_{i}$ converging to $F(v)$ in the flat norm. By the definition of mass, we may assume that each chain $C_{i}$ has mass less than $|F(v)|+\epsilon$. We have to show that we can choose the chains $C_{i}$ to be cycles. Because the boundary operation is continuous 
in the flat norm, we know that the flat norm of $\partial C_{i}$ converges to zero. By the definition of the flat norm, we may choose integral Lipschitz k-chains $D_{i}$ so that $\left|\partial C_{i}-\partial D_{i}\right|+\left|D_{i}\right| \rightarrow 0$. According to the isoperimetric inequality, for sufficiently large i, there is a k-chain $E_{i}$ with $\partial E_{i}=\partial C_{i}-\partial D_{i}$ and $\left|E_{i}\right|<C\left|\partial C_{i}-\partial D_{i}\right|$. Now we define $\tilde{C}_{i}=C_{i}-D_{i}-E_{i}$. Each $\tilde{C}_{i}$ is an integral Lipschitz k-cycle. Moreover, $\left|D_{i}\right|+\left|E_{i}\right| \rightarrow 0$. Since the mass controls the flat norm, $\tilde{C}_{i}$ converges to $F(v)$ in the flat topology. Since $\left|D_{i}\right|+\left|E_{i}\right| \rightarrow 0$, the mass of $\tilde{C}_{i}$ is less than $|F(v)|+2 \epsilon$ for sufficiently large $i$.)

Now, since the triangulation is fine, we may assume that if $v_{1}$ and $v_{2}$ are neighboring vertices, then the flat distance between $C\left(v_{1}\right)$ and $C\left(v_{2}\right)$ is less than $3 \delta$. By definition, this means that there is an integral Lipschitz $(k+1)$-chain $\mathrm{D}$ with $\left|C\left(v_{1}\right)-C\left(v_{2}\right)-\partial D\right|+|D|$ less than $3 \delta$. The k-cycle $C\left(v_{1}\right)-C\left(v_{2}\right)-\partial D$ must have mass less than $3 \delta$. According to the isoperimetric inequality, it must bound a $(\mathrm{k}+1)$ chain $D^{\prime}$ with mass at most $C(n) \delta$. In other words, $C\left(v_{1}\right)-C\left(v_{2}\right)=\partial\left(D+D^{\prime}\right)$. We know that the mass of $D+D^{\prime}$ is bounded by $C(n) \delta$.

Let $\mathrm{E}$ denote the edge from $v_{1}$ to $v_{2}$, oriented so that $\partial E=v_{1}-v_{2}$. We define $C(E)=D+D^{\prime}$. We repeat this operation for every edge of the triangulation of $\mathrm{P}$. For each edge $\mathrm{E}$ with boundary $v_{1}-v_{2}, C(E)$ is a $(\mathrm{k}+1)$-chain with boundary $C\left(v_{1}\right)-C\left(v_{2}\right)$ and mass at most $C(n) \delta$.

We continue this procedure inductively. For each oriented i-dimensional simplex $\Delta^{i}$ in $\mathrm{P}$, we define a $(\mathrm{k}+\mathrm{i})$-chain $C\left(\Delta^{i}\right)$ with the following properties. If the boundary of the simplex $\Delta^{i}$ is equal to $\sum_{j} \Delta_{j}^{i-1}$ with orientations, then the boundary of $C\left(\Delta^{i}\right)$ is equal to $\sum_{j} C\left(\Delta_{j}^{i-1}\right)$ as integral Lipschitz cycles. Moreover, $C\left(\Delta^{i}\right)$ has mass less than $C(n) \delta$. We can always choose such chains by using the isoperimetric inequality in $\mathrm{M}$, provided the initial number $\delta$ is sufficiently small.

The map $\mathrm{C}$ taking oriented simplices of $\mathrm{P}$ to integral Lipschitz chains in $\mathrm{M}$ can be thought of as a chain map between two chain complexes. The first complex is associated to P. To describe it, it is convenient to first pick an orientation for every simplex in $\mathrm{P}$. The complex has i-chains consisting of sums $c_{i} \Delta_{i}$, where $c_{i}$ is an integer and $\Delta_{i}$ is any i-dimensional simplex of $\mathrm{P}$. The homology of this chain complex is the simplicial homology of $\mathrm{P}$ with coefficients in $\mathbb{Z}$. The second chain complex consists of the integral Lipschitz chains in $\mathrm{M}$. The homology of this complex is equal to the singular homology of $\mathrm{M}$ with coefficients in $\mathbb{Z}$. The map $\mathrm{C}$ is a map from the first complex to the second complex, taking $i$-chains to $(k+i)$-chains, and commuting with boundary operations - in other words a chain map with shift $\mathrm{k}$. We call such a chain map $\mathrm{C}$ a complex of k-cycles in $\mathrm{M}$.

The chain map $\mathrm{C}$ induces a map from the simplicial homology of $\mathrm{P}$ to the singular homology of M. Since simplicial homology and singular homology agree, we get a map from $H_{i}(P, \mathbb{Z})$ to $H_{k+i}(M, \mathbb{Z})$. We call this map the gluing homomorphism $\mathrm{G}$.

The chain map $\mathrm{C}$ was not canonical. On the contrary it involved many choices. Nevertheless, the gluing homomorphism does not depend on these choices, as long as $\delta$ is sufficiently small. To see this, let $C_{0}$ and $C_{1}$ be two possible choices of chain map following the construction above. Divide $P \times[0,1]$ into cells given by $\Delta \times$ $\{0\}, \Delta \times\{1\}$, and $\Delta \times[0,1]$, where $\Delta$ varies over the triangulation of $\mathrm{P}$. Now define $C(\Delta \times\{0\})=C_{0}(\Delta)$ and $C(\Delta \times\{1\})=C_{1}(\Delta)$. Suppose that $\Delta^{i}$ is an i-simplex in $\mathrm{P}$, and that the boundary of $\Delta^{i} \times[0,1]$ is equal to $\Delta^{i} \times\{1\}-\Delta^{i} \times\{0\}+\sum_{j} \Delta_{j}^{i-1} \times[0,1]$ with orientations. Proceeding inductively, we define $C\left(\Delta^{i} \times[0,1]\right)$ to be a $(\mathrm{k}+\mathrm{i}+1)$ chain with boundary $C\left(\Delta^{i} \times\{1\}\right)-C\left(\Delta^{i} \times\{0\}\right)+\sum_{j} C\left(\Delta_{j}^{i-1} \times[0,1]\right)$. Again, 
as long as we assume $\delta$ sufficiently small, we can construct all these chains using the isoperimetric inequality. We can view $\mathrm{C}$ as a chain map as well, and it induces a map on homology from $H_{i}(P \times[0,1], \mathbb{Z})$ to $H_{k+i}(M, \mathbb{Z})$. This map agrees with the gluing homomorphisms induced by both $C_{0}$ and $C_{1}$, and so these two gluing homomorphisms agree with one another.

We say that a family of k-cycles $\mathrm{F}$ sweeps out $\mathrm{M}$ if its gluing homomorphism $G: H_{n-k}(P, \mathbb{Z}) \rightarrow H_{n}(M, \mathbb{Z})$ is non-trivial.

We define the $\mathrm{k}$-width of $\mathrm{M}$ to be the infimal width of any family of k-cycles sweeping out $\mathrm{M}$. We denote the k-width of $\mathrm{M}$ by $W_{k}(M)$.

(On a manifold with boundary, the $\mathrm{k}$-width is defined using relative flat $\mathrm{k}$-cycles. The above arguments also apply to relative chains and cycles. In this case, the gluing homomorphism maps $H_{i}(P, \mathbb{Z})$ to $H_{k+i}(M, \partial M, \mathbb{Z})$. We say that a family $\mathrm{F}$ of relative k-cycles sweeps out $\mathrm{M}$ if the gluing homomorphism from $H_{n-k}(P)$ to $H_{n}(M, \partial M)$ is non-trivial.)

Next we discuss some basic properties of the k-width.

\section{Monotonicity}

The k-width is monotonic in two respects. First, if $\mathrm{M}$ is an open subset of $(N, g)$ with the induced metric, then the $\mathrm{k}$-width of $\mathrm{M}$ is at most the $\mathrm{k}$-width of $\mathrm{N}$. Let $\mathrm{F}$ be a family of k-cycles sweeping out $\mathrm{N}$ with width less than $W_{k}(N)+\epsilon$. There is a restriction map which takes integral Lipschitz chains in $\mathrm{N}$ to integral Lipschitz chains in M. This map commutes with the boundary action and is bounded in the flat norm. Therefore, it takes flat k-cycles in $\mathrm{N}$ to (relative) flat k-cycles in $\mathrm{M}$. Restricting $\mathrm{F}$ to $\mathrm{M}$ gives a family of cycles sweeping out $\mathrm{M}$ with width less than $W_{k}(N)+\epsilon$. This proves the first form of monotonicity.

The second form of monotonicity concerns maps from $\mathrm{M}$ to $\mathrm{N}$. If $\mathrm{f}$ is a Lipschitz map between compact Riemannian manifolds $\mathrm{M}$ and $\mathrm{N}$, then it maps integral Lipschitz chains in M to integral Lipschitz chains in N. This induced map is continous with respect to the flat distance, so it maps flat k-chains in $\mathrm{M}$ to flat $\mathrm{k}$-chains in $\mathrm{N}$. If $\mathrm{M}$ and $\mathrm{N}$ are both closed, it maps flat k-cycles in $\mathrm{M}$ to flat k-cycles in $\mathrm{N}$. If $\mathrm{M}$ and $\mathrm{N}$ both have boundaries, and if $\mathrm{f}$ maps $(M, \partial M)$ to $(N, \partial N)$, then $\mathrm{f}$ maps relative flat k-cycles in $\mathrm{M}$ to relative flat k-cycles in $\mathrm{N}$. If $\mathrm{M}$ has boundary and $\mathrm{N}$ is closed, and if $\mathrm{f}$ maps $(M, \partial M)$ to $(N, *)$, where $*$ is a point of $\mathrm{N}$, then $\mathrm{f}$ takes relative flat $\mathrm{k}$-cycles in $\mathrm{M}$ to flat $\mathrm{k}$-cycles in $\mathrm{N}$. The construction above shows that the gluing homomorphism is natural.

If $\mathrm{f}$ is a Lipschitz map from $\mathrm{M}$ to $\mathrm{N}$ with Lipschitz constant $\mathrm{L}$ and non-zero degree then $L^{k} W_{k}(M) \geq W_{k}(N)$. To see this, let $\mathrm{F}$ be a family of flat k-cycles sweeping out $\mathrm{M}$ with width less than $W_{k}(M)+\epsilon$. Since $\mathrm{f}$ has non-zero degree, the image $f(F)$ is a family of k-cycles sweeping out N. Since $\mathrm{f}$ maps each integral Lipschitz k-chain with mass $\mathrm{M}$ to one with mass at most $L^{k} M$, the width of $f(F)$ is less than $L^{k}\left(W_{k}(M)+\epsilon\right)$.

The last estimate really only depended on how much the map f stretched kdimensional volumes. Let $\mathrm{f}$ be a piecewise smooth map. Recall that the k-dilation of $f$ is at most $\lambda$ if $f$ maps each $\mathrm{k}$-dimensional submanifold of the domain with volume $\mathrm{V}$ to an image with volume at most $\lambda V$. For more information on k-dilation, see the beginning of section 3 . Let $\mathrm{f}$ be a piecewise smooth map of non-zero degree from $\mathrm{M}$ to $\mathrm{N}$ with k-dilation $\lambda$. Since Lipschitz maps can be well-approximated by $C^{1}$ maps, $\mathrm{f}$ takes each integral Lipschitz chain with mass $\mathrm{M}$ to an integral Lipschitz chain with mass at most $\lambda M$. Let $\mathrm{F}$ be a family of $\mathrm{k}$-cycles sweeping out $\mathrm{M}$ with width at most 
$W_{k}(M)+\epsilon$. Then $\mathrm{f}(\mathrm{F})$ sweeps out $\mathrm{N}$ with width at most $\lambda\left(W_{k}(M)+\epsilon\right)$. Therefore, $\lambda W_{k}(M) \geq W_{k}(N)$.

\section{Non-degeneracy}

The non-degeneracy property says that $W_{k}(M)>0$ for any $(M, g)$. Because of the monotonicity estimates for $W_{k}$, the non-degeneracy follows for every n-manifold as soon as we know that $W_{k}\left(S^{n}\right)>0$ for the standard round metric on $S^{n}$. Gromov gave an elegant elementary proof of this fact which we include here in our language. This proof originally appeared in [7].

Proposition 1.1. (Gromov) For the standard round metric on $S^{n}$, the k-width $W_{k}\left(S^{n}\right)$ is greater than $c(n)>0$.

Proof. Suppose not. Then there is a family $\mathrm{F}$ of k-cycles sweeping out $S^{n}$ with width less than $\epsilon$. Above, we constructed a complex of cycles $\mathrm{C}$ based on $\mathrm{F}$, with a non-trivial gluing map. For each vertex $\mathrm{v}$ of the triangulation of the parameter space $\mathrm{P}$, we had $|C(v)|<\epsilon+\delta$, and for each higher dimensional simplex $\Delta^{i}$ of $\mathrm{P}$, we had $\left|C\left(\Delta^{i}\right)\right|<C(n) \delta$. These estimates hold for a number $\delta$ as small as we like. We assume $\delta$ much smaller than $\epsilon$.

Using the isoperimetric inequality, each cycle $C(v)$ can be filled by a $(\mathrm{k}+1)$ chain of mass less than $C(n) \epsilon$. For each vertex v of the triangulation, define Fill $(v)$ to be such a filling. Now let $\mathrm{E}$ be an oriented edge of the triangulation with $\partial E=v_{1}-v_{2}$. We define $\bar{C}(E)=C(E)-\operatorname{Fill}\left(v_{1}\right)+F i l l\left(v_{2}\right)$. Since the boundary of Fill $\left(v_{i}\right)=C\left(v_{i}\right), \bar{C}(E)$ is a $(\mathrm{k}+1)$-cycle. The mass of $\bar{C}(E)$ is bounded by $C(n) \epsilon$. Next, using the isoperimetric inequality again, choose an oriented $(\mathrm{k}+2)$ chain Fill $(E)$ with boundary $\bar{C}(E)$, and with mass bounded by $C(n) \epsilon$.

We then repeat this construction for the higher-dimensional simplices in the triangulation of $\mathrm{P}$, working one skeleton at a time. For each i-simplex $\Delta^{i}$ of $\mathrm{P}$, we define a $(\mathrm{k}+\mathrm{i})$-cycle $\bar{C}\left(\Delta^{i}\right)$ and a $(k+i+1)$-chain $\operatorname{Fill}\left(\Delta^{i}\right)$. They have the following properties.

1. If $\Delta^{i}$ is an i-simplex of $\mathrm{P}$, and the boundary of $\Delta^{i}=\sum_{j} \Delta_{j}^{i-1}$, then

$\bar{C}\left(\Delta^{i}\right)=C\left(\Delta^{i}\right)-\sum_{j} \operatorname{Fill}\left(\Delta_{j}^{i-1}\right)$.

2. If $\sum_{l} c_{l} \Delta_{l}^{i}$ is any $\mathrm{i}$-cycle in $\mathrm{P}$, then

$\sum_{l} c_{l} C\left(\Delta_{l}^{i}\right)=\sum c_{l} \bar{C}\left(\Delta_{l}^{i}\right)$.

3. The boundary of Fill $\left(\Delta^{i}\right)=\bar{C}\left(\Delta^{i}\right)$.

4. The mass of each $C\left(\Delta^{i}\right)$ and each Fill $\left(\Delta^{i}\right)$ is bounded by $C(n) \epsilon$.

To prove that we can find $\bar{C}\left(\Delta^{i}\right)$ and $F i l l\left(\Delta^{i}\right)$ we work inductively. We already did the case $i=1$, which anchors the induction. We assume that the above properties hold for simplices of dimension at most i-1. We define $\bar{C}\left(\Delta^{i}\right)$ by using the formula in 1 . We have to check that $\bar{C}\left(\Delta^{i}\right)$ is a cycle. Its boundary is $\sum_{j} C\left(\Delta_{j}^{i-1}\right)-\sum_{j} \bar{C}\left(\Delta_{j}^{i-1}\right)$. According to the equation in 2 and the inductive hypothesis, this expression vanishes. Next, we have to check that $\bar{C}$ obeys equation 2 for i-simplices. Let $\sum_{l} c_{l} \Delta_{l}^{i}$ be an i-cycle in P. Let the boundary of $\Delta_{l}^{i}$ be $\sum_{j} \Delta_{l, j}^{i-1}$. Because $\sum_{l} c_{l} \Delta_{l}^{i}$ is an i-cycle, $\sum_{l, j} c_{l} \Delta_{l, j}^{i-1}=0$. Now $\sum_{l} c_{l} \bar{C}\left(\Delta_{l}^{i}\right)=\sum_{l}\left(c_{l} C\left(\Delta_{l}^{i}\right)+c_{l} \sum_{j} \operatorname{Fill}\left(\Delta_{l, j}^{i-1}\right)\right)$, and the terms in the second sum cancel because $\sum_{l} c_{l} \Delta_{l}^{i}$ is a cycle. Therefore, $\bar{C}$ obeys equation 2 for i-simplices. Because $C\left(\Delta^{i}\right)$ has mass at most $\delta$ and $F i l l\left(\Delta_{j}^{i-1}\right)$ has mass at most $C(n) \epsilon, \bar{C}\left(\Delta^{i}\right)$ has mass at most $C(n) \epsilon$. Therefore, we can use the isoperimetric inequality to define $\operatorname{Fill}\left(\Delta^{i}\right)$ with mass at most $C(n) \epsilon$. 
Since F sweeps out $S^{n}$, there must be an (n-k)-cycle $a$ in $\mathrm{P}$ with $G(a)$ non-trivial in $H_{n}\left(S^{n}, \mathbb{Z}\right)$. Write $a=\sum c_{m} \Delta_{m}^{n-k}$. By definition $\sum c_{m} C\left(\Delta_{m}^{n-k}\right)$ has a non-trivial homology class in $H_{n}\left(S^{n}, \mathbb{Z}\right)$. But this sum is equal to a sum of cycles $\sum c_{m} \bar{C}\left(\Delta_{m}^{i}\right)$. Each cycle $\bar{C}\left(\Delta_{m}^{i}\right)$ has mass less than $C(n) \epsilon$, and hence is null-homologous in $S^{n}$. This contradiction finishes the proof.

We now estimate the k-widths of some simple shapes. The k-width of the unit $\mathrm{n}$-cube is at most 1 , because it is swept out by parallel $\mathrm{k}$-planes each meeting it in a unit k-cube. Because of the non-degeneracy proposition and the monotonicity estimate, the $\mathrm{k}$-width of the unit $\mathrm{n}$-cube is at least $c(n)>0$.

Applying the monotonicity estimate to our bounds for the unit cube, we can estimate the k-width of any rectangle. Let $R$ be a rectangle with dimensions $R_{1} \leq$ $\ldots \leq R_{n}$. In other words, $\mathrm{R}$ is the product $\left[0, R_{1}\right] \times \ldots \times\left[0, R_{n}\right]$.

Proposition 1.2. The $k$-width of the rectangle $R$ is roughly $R_{1} \ldots R_{k}$. More precisely, $c(n) R_{1} \ldots R_{k} \leq W_{k}(R) \leq R_{1} \ldots R_{k}$.

Proof. To get the upper bound, simply consider the projection of $\mathrm{R}$ onto the last $(\mathrm{n}-\mathrm{k})$ coordinates. Each fiber of this projection is a k-dimensional rectangle with volume $\left(R_{1} \ldots R_{k}\right)$, and the fibers fit together to form a family of k-cycles sweeping out $\mathrm{R}$.

To get the lower bound, consider the map from $R$ to the unit cube which sends $\left(x_{1}, \ldots, x_{n}\right)$ to $\left(x_{1} / R_{1}, \ldots, x_{n} / R_{n}\right)$. This map has degree 1 and k-dilation $\left(R_{1} \ldots R_{k}\right)^{-1}$. Therefore $\left(R_{1} \ldots R_{k}\right)^{-1} W_{k}(R) \geq W_{k}(C)$, where $\mathrm{C}$ denotes the unit cube. According to Proposition 1.1, $W_{k}(C) \geq c(n)>0$, so $W_{k}(R) \geq c(n) R_{1} \ldots R_{k}$.

\section{The WIDTh-VOLUme INEQUALITY}

In this section, we prove Theorem 1.

Theorem 1. (Width-volume inequality) Let $U$ be a bounded open set in $\mathbb{R}^{n}$ with volume $V(U)$ and $k$-width $W_{k}(U)$. Then $W_{k}(U)<C(n) V(U)^{k / n}$.

Proof. By a scaling argument, it suffices to prove the theorem when the volume of $U$ is 1 .

The first step in the proof is to translate the unit lattice so that its k-skeleton meets $U$ in a region of controlled volume. Let $S(x)$ denote the k-skeleton of the unit cubical lattice centered at $\mathrm{x}$, with axes parallel to the coordinates. Since the volume of $U$ is 1 , the average volume of $U \cap S(x)$ as x varies over the unit cube is equal to $\left(\begin{array}{l}n \\ k\end{array}\right)$. We can choose a point $\mathrm{x}$ so that the volume of $U \cap S(x)$ is no more than the average value $\left(\begin{array}{l}n \\ k\end{array}\right)$. From now on, we refer to $\mathrm{S}(\mathrm{x})$ simply as $\mathrm{S}$.

The second step in the proof is to construct a family of cycles sweeping out $\mathrm{U}$, each of which lies mostly in the k-skeleton S.

Construction 1. (Bending planes around a skeleton) Let $B(R)$ denote the ball of radius $R$ in $\mathbb{R}^{n}$, and let $S$ be the k-skeleton of a unit lattice. Then there is a family $F$ of $k$-cycles sweeping out $B(R)$ with the following properties. Each cycle in $F$ lies in $S$ except for a subset of mass less than $C(n)$. A cycle in $F$ may contain some portions of $S$ with multiplicity greater than 1, but this multiplicity is bounded by $C(n)$. (The constant $C(n)$ depends only on $n$; it does not depend on the radius $R$. ) 
Using this family of k-cycles we finish the proof of Theorem 1. By choosing $R$ sufficiently large, we may assume that $U$ lies in the ball $B(R)$. Then we consider the restriction of $\mathrm{F}$ to $\mathrm{U}$, which is a family of $\mathrm{k}$-cycles sweeping out $\mathrm{U}$. To prove Theorem 1, we only have to check that each k-cycle in this family has mass at most $C(n)$. Let $E$ be a k-cycle in F. In other words, we have to check that the mass of E restricted to $U$ is at most $C(n)$. We divide the restriction of $E$ to $U$ into two pieces. We let $E_{1}$ be the part of this restriction which is contained in $\mathrm{S}$, and we let $E_{2}$ be the part of this restriction which is not contained in $\mathrm{S}$. The chain $E_{1}$ is contained in $\mathrm{S}$, and according to Construction 1 , it has multiplicity at most $C(n)$. Therefore its mass is at most $C(n)|S \cap U| \leq\left(\begin{array}{l}n \\ k\end{array}\right) C(n)$. On the other hand, according to Construction 1, $E_{2}$ has mass at most $C(n)$.

Next we turn to the proof of Construction 1.

Proof. We begin with a family of parallel k-planes. Let $\mathrm{P}$ be an $(n-k)$-plane through the origin, in general position with respect to $\mathrm{S}$. Let $F_{0}$ be the family of all k-planes perpendicular to $\mathrm{P}$. To bend the planes, we will construct a degree 1 proper PL map $\Psi$ from $\mathbb{R}^{n}$ to itself. Our family $\mathrm{F}$ will be $\Psi\left(F_{0}\right)$. In other words, the cycles in $\mathrm{F}$ will be $\Psi(Q)$ as $\mathrm{Q}$ varies over all the k-planes perpendicular to $\mathrm{P}$. Because $\Psi$ is degree 1 , this family of cycles sweeps out the ball $B(R)$ or any other open set.

The reader can roughly imagine $\Psi$ as follows. Let $\mathrm{T}$ denote the dual $(n-k-1)$ skeleton to $\mathrm{S}$, and let $T_{\epsilon}$ denote the $\epsilon$-neighborhood of T. The mapping $\Psi$ retracts the complement of $T_{\epsilon}$ onto $S$ while stretching $T_{\epsilon}$ to fill all of $\mathbb{R}^{n}-S$.

The idea of our proof is as follows. Since $\Psi$ retracts the complement of $T_{\epsilon}$ onto S, the map $\Psi$ takes $Q \cap\left(\mathbb{R}^{n}-T_{\epsilon}\right)$ into S. On the other hand, we will try to control the size of $Q \cap T_{\epsilon}$ using the fact that $\mathrm{T}$ is $(n-k-1)$-dimensional and $\mathrm{Q}$ is $k$-dimensional. Because of these dimensions, a generic plane $\mathrm{Q}$ will not intersect $\mathrm{T}$ at all. The set of planes $\mathrm{Q}$ in $F_{0}$ which intersect $\mathrm{T}$ has codimension 1 . The set of planes which intersect $\mathrm{T}$ twice has codimension 2, and so on. Therefore, each plane $\mathrm{Q}$ intersects $\mathrm{T}$ at most $(n-k)$ times. Using this kind of argument, we will show that $Q \cap T_{\epsilon}$ is contained in a union of $(n-k)$ small balls. Finally we will have to analyze the action of $\Psi$ on each of these small balls. This last step requires us to write down the map $\Psi$ carefully.

We state the properties of the map $\Psi$ we will use in the form of a lemma.

Lemma 2.1. For each $\epsilon>0$, there is a piecewise-linear map $\Psi$ from $\mathbb{R}^{n}$ to itself with the following properties. The map $\Psi$ is linear on each simplex of a certain triangulation of $\mathbb{R}^{n}$. Each top-dimensional simplex of this triangulation is labelled good or bad. For each good simplex $\Delta, \Psi(\Delta)$ lies in $S$. Each bad simplex lies in $T_{\epsilon}$. The triangulation and the map obey the following bounds.

1. The number of simplices of our triangulation meeting any unit ball is bounded by $C(n)$.

2. The displacement $|\Psi(x)-x|$ is bounded by $C(n)$.

3. The diameter of each simplex is bounded by $C(n)$.

The only tricky part in checking this lemma is to get the bounds with constants that don't depend on $\epsilon$. We defer the proof of the lemma until we finish the construction.

Because of the displacement bound, the map $\Psi$ is proper. We can deform $\Psi$ to the identity by taking $\Psi_{t}(x)=(1-t) \Psi(x)+t x$. Then $\Psi_{0}$ is equal to $\Psi$ and $\Psi_{1}$ 
is the identity. Each map $\Psi_{t}$ also obeys the displacement bound, so they are all proper. Therefore, $\Psi$ has degree 1.

We think of the cycle $\Psi(Q)$ as a sum of chains $\sum \Psi(Q \cap \Delta)$ as $\Delta$ varies over all the simplices of our triangulation.

We first consider the contribution to $\Psi(Q)$ coming from the good simplices. For each good simplex $\Delta, \Psi(\Delta)$ lies in $S$, and so $\Psi(Q \cap \Delta)$ also lies in $S$. Since $\Psi$ is linear on $\Delta$, the image $\Psi(Q \cap \Delta)$ has multiplicity at most 1. Next, we bound the multiplicity of the sum $\sum_{\Delta} \Psi(Q \cap \Delta)$ as $\Delta$ ranges over all the good simplices. Because of the displacement bound in Lemma 2.1, the multiplicity of this sum at a point $s$ in $S$ only depends on the contributions from good simplices $\Delta$ in a ball around $s$ of radius $C(n)$. But estimate 1 in Lemma 2.1 tells us that there are less than $C(n)$ simplices in this ball.

We now consider the contribution to $\Psi(Q)$ coming from the bad simplices. Since we are only proving bounds for the restriction of $\Psi(Q)$ to the ball $B(R)$, we only need to consider the bad simplices $\Delta$ so that $\Psi(\Delta)$ intersects the ball $B(R)$. Because of the displacement bound in Lemma 2.1, we only need to consider the bad simplices $\Delta$ in the ball of radius $R+C(n)$. We let $B\left(R^{\prime}\right)$ denote this larger ball, and from now on we only consider the bad simplices in this ball. This argument about balls is not the main point, but it is technically easier to proceed this way because we only have to consider finitely many simplices.

Next we show that if $\epsilon$ is sufficiently small, the plane Q intersects less than $C(n)$ bad simplices. This estimate is the main idea of the proof. Recall that $\mathrm{Q}$ is a plane perpendicular to the (n-k)-plane P. Let $\pi$ denote the orthogonal projection from $\mathbb{R}^{n}$ onto P. The plane $\mathrm{Q}$ is one of the fibers of $\pi$. Let $T^{\prime}$ denote the finite complex $T \cap B\left(R^{\prime}\right)$. Note that $T^{\prime}$ is contained in a finite union of $(n-k-1)$-planes. We denote these planes as $T_{i}$. Since $\mathrm{P}$ is in general position with respect to $\mathrm{T}$, the projections $\pi\left(T_{i}\right)$ are a finite set of $(n-k-1)$-planes in $\mathrm{P}$, meeting transversely. Therefore, any point $p$ in $\mathrm{P}$ lies in $\pi\left(T_{i}\right)$ for at most $(n-k)$ values of $i$. Since the number of planes is finite, we can choose $\epsilon$ sufficiently small so that any point $p$ in $P$ lies within $\epsilon$ of $\pi\left(T_{i}\right)$ for at most $(n-k)$ values of $i$. Since $Q$ is a fiber of $\pi$, it meets the $\epsilon$-neighborhood of $T_{i}$ for at most $(n-k)$ values of $i$. Since $Q$ is transverse to each of these planes, $Q \cap T_{\epsilon}^{\prime}$ is contained in $(n-k)$ balls each of radius $C(n) \epsilon$. Because of estimate 1 in Lemma 2.1, these balls meet at most $C(n)$ bad simplices, and so $Q$ intersects at most $C(n)$ bad simplices.

Finally, we bound the volume of $\Psi(Q \cap \Delta)$ where $\Delta$ is a bad simplex. Because of the diameter bound and the displacement bound, $\Psi(\Delta)$ has diameter at most $C(n)$. Therefore, $\Psi(Q \cap \Delta)$ is a portion of k-plane with diameter at most $C(n)$. We conclude that $\Psi(Q \cap \Delta)$ has volume at most $C(n)$.

We finish the proof of Construction 1 by assembling these estimates. The cycle $\Psi(Q)$ lies in $S$ except for the contributions from the bad simplices. There are at most $C(n)$ bad simplices, and each bad simplex contributes mass at most $C(n)$, and so the cycle $\Psi(Q)$ lies in $S$ except for a portion with mass at most $C(n)$. The multiplicity of $\Psi(Q)$ is also bounded. We already bounded the contribution to the multiplicity coming from the good simplices. Since Q intersects only $C(n)$ bad simplices in $B\left(R^{\prime}\right)$, the contribution to the multiplicity coming from the bad simplices is also bounded.

Before we go on, let us clarify which constants depend on which other constants. The most important point is that the constants $C(n)$ depend only on $n$. The 
constants $C(n)$ don't depend on R. On the other hand, the size of $\epsilon$ that we need to make the above construction work does depend on $\mathrm{R}$. Therefore, we need to prove Lemma 2.1 with constants $C(n)$ that don't depend on $\epsilon$. We now give the proof of Lemma 2.1.

Proof. We will now construct the map $\Psi$. We begin by constructing the triangulation of good and bad simplices. First we need to make some definitions. If $\mathrm{A}$ is a $\mathrm{k}$-dimensional face in $\mathrm{S}$, then we define the link of $\mathrm{A}$ in the following way. The set $\mathrm{A}$ is defined by equations $x_{i}=a_{i}$ for (n-k) coordinates $\mathrm{i}$, and equations $a_{j} \leq x_{j} \leq a_{j}+1$ for the other $\mathrm{k}$ coordinates. There is an (n-k) cube transverse to A given by the equations $a_{i}-1 / 2 \leq x_{i} \leq a_{i}+1 / 2$ for the (n-k) coordinates $\mathrm{i}$ above, and $x_{j}=a_{j}+1 / 2$ for the other $\mathrm{k}$ coordinates. This cube is simply the (n-k) cube centered at the center of A, perpendicular to A, with axes parallel to the coordinate axes. The link of A is defined to be the boundary of this (n-k)-cube. It consists of $2(n-k)(n-k-1)$-cubes, each of which is an (n-k-1)-dimensional face of $\mathrm{T}$. If $\mathrm{B}$ is an (n-k-1) dimensional face of $\mathrm{T}$, we define the link of $\mathrm{B}$ in an analogous way. It is a topological $\mathrm{k}$-sphere consisting of $2(\mathrm{k}+1) \mathrm{k}$-dimensional faces of $\mathrm{S}$. We let A denote a k-dimensional face of $S$ and B an (n-k-1)-dimensional face of T. A quick calculation shows that $\mathrm{A}$ is in the link of $\mathrm{B}$ if and only if $\mathrm{B}$ is in the link of A. For each pair $(\mathrm{A}, \mathrm{B})$ of faces with $\mathrm{A}$ in the link of $\mathrm{B}$, we define $K(A, B)$ to be the convex hull of the union of $\mathrm{A}$ and $\mathrm{B}$.

Next we check that the sets $K(A, B)$ tile $\mathbb{R}^{n}$. The hyperfaces of the tile $K(A, B)$ correspond to pairs $(A, b)$ where $\mathrm{b}$ is an (n-k-2)-face in the boundary of $\mathrm{B}$, or pairs $(a, B)$, where a is a (k-1)-face in the boundary of A. (The corresponding face is just the convex hull of $\mathrm{A}$ and $\mathrm{b}$, or of $\mathrm{a}$ and $\mathrm{B}$.) Each face borders exactly two tiles in our tiling. Given a face $(A, b)$, let $B^{\prime}$ be the (n-k-1)-face in the link of $\mathrm{A}$ which lies on the other side of $\mathrm{b}$ from $\mathrm{B}$. Then $K\left(A, B^{\prime}\right)$ is the only other tile with $(A, b)$ as a face. Therefore, the tiles form a pseudo-manifold, and the embedding of the tiles is an orientation preserving proper map from the tile space to $\mathbb{R}^{n}$. The intersection of $K(A, B)$ with the skeleton $\mathrm{S}$ is equal to $\mathrm{A}$. In particular, the only tiles that come near to the center of $\mathrm{A}$ are tiles $K(A, B)$ for some $\mathrm{B}$ in the link of $\mathrm{A}$. It is easy to check that a typical point close to the center of A lies in exactly one of the tiles $K(A, B)$. Therefore, the tiles have disjoint interiors and cover all of space.

Any two tiles in our tiling are isometric. After renumbering the coordinates, translating, and reflecting, we can assume that $\mathrm{A}$ and $\mathrm{B}$ have the following simple form. The face $\mathrm{A}$ is given by the inequalities $0 \leq x_{i} \leq 1$ for $\mathrm{i}$ from 1 to $\mathrm{k}, x_{i}=0$ for $\mathrm{i}$ from $k+1$ to $n$. The face $\mathrm{B}$ is given by inequalities $-1 / 2 \leq x_{i} \leq 1 / 2$ for $\mathrm{i}$ from $k+1$ to $n-1$, the equations $x_{i}=1 / 2$ for $\mathrm{i}$ from 1 to $\mathrm{k}$, and $x_{n}=1 / 2$. The convex set $K(A, B)$ is given by the inequalities $0 \leq x_{n} \leq 1 / 2,-x_{n} \leq x_{i} \leq x_{n}$ for $\mathrm{i}$ from $k+1$ to $n-1$, and $\left|1 / 2-x_{i}\right| \leq\left|1 / 2-x_{n}\right|$ for i from 1 to $k$.

We now divide each tile $K(A, B)$ into good and bad parts. The good part of $K(A, B)$ is given by $x_{n} \leq 1 / 2-\epsilon$ and denoted $K_{G}(A, B)$. The bad part of $K(A, B)$ is given by $x_{n} \geq 1 / 2-\epsilon$ and denoted by $K_{B}(A, B)$. In other words, the bad part of $K(A, B)$ lies in a small neighborhood of $\mathrm{B}$, and its complement is the good part. Since B is a face of $\mathrm{T}$, the bad part of $K(A, B)$ lies in $C(n) \epsilon$ neighborhood of $\mathrm{T}$.

If $\mathrm{K}$ is any convex polyhedron, we can define a barycentric triangulation for $\mathrm{K}$ as follows. For each face $\mathrm{F}$ of $\mathrm{K}$, of any dimension, let $c(F)$ denote the center of mass of $F$. The triangulation of the 0 -skeleton of $\mathrm{K}$ is trivial. Now suppose we have triangulated the $\mathrm{i}$-skeleton of $\mathrm{K}$. We extend this triangulation to each $(i+1)$-face 
$\mathrm{F}$ of $\mathrm{K}$, by taking the cone from $c(F)$ to the triangulation on the boundary of $\mathrm{F}$. A good thing about the barycentric triangulation is that if two convex polyhedra intersect in a face of any dimension, then the two barycentric sub-divisions of that face agree. Therefore, applying the barycentric subdivision to each good and bad polyhedron in our tiling, we get a triangulation of $\mathbb{R}^{n}$. This is the triangulation that appears in the statement of the lemma. The map $\Psi$ will be linear on each simplex of this triangulation. We call a top-dimensional simplex good if it lies in the good part of $K(A, B)$ and bad if it lies in the bad part of $K(A, B)$.

At this point, we can check some of the bounds in the lemma. The number of simplices in a unit ball is bounded by $C(n)$. The number of tiles $K(A, B)$ does not depend on $\epsilon$ at all. The combinatorial structure of the tiles $K_{G}(A, B)$ and $K_{B}(A, B)$ also does not depend on $\epsilon$. Therefore, the number of simplices in the barycentric triangulation also does not depend on $\epsilon$. Each simplex in contained in some set $K(A, B)$ and so has diameter at most $C(n)$. Also, each bad simplex lies within a $C(n) \epsilon$-neighborhood of $\mathrm{T}$.

To finish the proof of the Lemma, we need to construct the map $\Psi$. We will have to check that $\Psi$ is linear on each simplex of our triangulation, that $\Psi$ maps each good simplex into $\mathrm{S}$, and that $\Psi$ obeys the displacement bound.

The map $\Psi$ will take $K_{G}(A, B)$ onto $\mathrm{A}$ and $K_{B}(A, B)$ onto $K(A, B)$. We will specify the value of $\Psi$ at the center of each face of $K_{G}(A, B)$ and of $K_{B}(A, B)$. We then define $\Psi$ to be the unique function which is linear on the barycentric subdivision and takes these values at the centers of the faces. To carry this out, we must write down all of the faces in $K_{G}(A, B)$ and $K_{B}(A, B)$.

The faces in $K(A, B)$ are as follows. First, any face a of A. Second, any face $\mathrm{b}$ of B. Third, the convex hull of any face a of A and any face b of B. (These faces may have any dimensions.)

The faces of $K_{G}(A, B)$ are as follows. First, any face a of $\mathrm{A}$. Second, the intersection of the convex hull of a and $\mathrm{b}$ with the set $x_{n} \leq 1 / 2-\epsilon$. Third, the intersection of the convex hull of a and $\mathrm{b}$ with the set $x_{n}=1 / 2-\epsilon$. In each case, we define $\Psi(c(F))$ to be the center of a. Therefore, $\Psi$ maps $K_{G}(A, B)$ into A.

The faces of $K_{B}(A, B)$ are as follows. First, any face b of B. Second, the intersection of the convex hull of a and $\mathrm{b}$ with the set $x_{n} \geq 1 / 2-\epsilon$. Third, the intersection of the convex hull of a and $\mathrm{b}$ with the set $x_{n}=1 / 2-\epsilon$. In the first case, we define $\Psi(c(F))$ to be the center of b. In the last two cases, we define $\Psi(c(F))$ to be the center of a.

If a certain face $\mathrm{F}$ belongs to several different polyhedra, then we have to check that our definition for $\Psi(c(F))$ is consistent. For this purpose, it suffices to check that the faces $\mathrm{a}$ and $\mathrm{b}$ are defined consistently. The face $\mathrm{b}$ is recovered as the largest face of $\mathrm{T}$ in the $n \epsilon$ neighborhood of $\mathrm{F}$, provided $\epsilon$ is sufficiently small. The face a is recovered as the smallest face of $S$ so that $\mathrm{F}$ is contained in the convex hull of a and $b$.

From the construction, we see that $\Psi$ is linear on each simplex of our triangulation. If $\Delta$ is a good simplex, then each vertex of $\Delta$ corresponds to the center of a face of $K_{G}(A, B)$, and so it gets mapped to a point in A. Since A is convex, the simplex $\Delta$ is mapped into A, and so $\Psi(\Delta)$ lies in S. Finally, if $\Delta$ denotes any good or bad simplex in $K(A, B)$, then $\Psi(\Delta)$ lies in $K(A, B)$, and so $\Psi$ obeys the displacement bound. 
There is an analogue of Theorem 1 for the widths of functions instead of sets. Once we define the k-width of a function, the proof is exactly the same. Let $f$ be a compactly supported function on $\mathbb{R}^{n}$ which is greater than or equal to zero. Let $B(R)$ denote a large ball containing the support of $\mathrm{f}$. If $\mathrm{F}$ is a family of k-cycles in $B(R)$, then we define the k-width of $\mathrm{F}$ to be the supremum of $\int_{F(p)} f$ over all $\mathrm{p}$ in the parameter space of $\mathrm{F}$. We define the $\mathrm{k}$-width of $\mathrm{f}$ to be the infimal $\mathrm{W}$ so that there is a family of $\mathrm{k}$-cycles sweeping out $B(R)$ with $\mathrm{k}$-width less than $\mathrm{W}$. The $\mathrm{k}$-width of $\mathrm{f}$ is denoted $W_{k}(f)$.

Proposition 2.1. If $f$ is a function with compact support on $\mathbb{R}^{n}$, and $0 \leq f \leq 1$, then $W_{k}(f)<C(n)\left(\int f\right)^{k / n}$.

Proof. After rescaling the coordinates, it suffices to prove that $W_{k}(f)<C(n)$ when $\int f=1$.

By translating the k-skeleton of the unit lattice, we can arrange that $\int_{S} f \leq$ $\left(\begin{array}{l}n \\ k\end{array}\right)$. Next we apply Construction 1, bending planes around the skeleton $\mathrm{S}$. This construction gives us a family $\mathrm{F}$ of k-cycles sweeping out $B(R)$. To prove the proposition, we have to bound the integral $\int_{E} f$, where $E$ is a k-cycle in the family F. We define $E_{1}$ to be the part of $E$ which is contained in $S$, and we define $E_{2}$ to be the part of $E$ which is not contained in $\mathrm{S}$. The chain $E_{1}$ is contained in $S$ and, according to Construction 1 , it has multiplicity at most $C(n)$. Therefore, $\int_{E_{1}} f \leq C(n) \int_{S} f \leq C(n)\left(\begin{array}{l}n \\ k\end{array}\right)$. On the other hand, $E_{2}$ has mass at most $C(n)$. Because $0 \leq f \leq 1$, we have the bound $\int_{E_{2}} f \leq C(n)$.

In our applications, we will need a width-volume inequality adapted to subsets of rectangles. Let $R$ be the n-dimensional rectangle with dimensions $R_{1} \leq$ $\ldots \leq R_{n}$. Let $U$ be a subset of $R$ with volume $V(U)$. What is the largest possible k-width of such a set $U$ ? We already know that $W_{k}(U)<C V(U)^{k / n}$, but this estimate turns out not to be sharp. A round ball with volume $V(U)$ has k-width roughly $V(U)^{k / n}$. If $V(U)^{1 / n}$ is much larger than $R_{1}$, the round ball with volume $V(U)$ does not fit inside of the rectangle $R$. What are the subsets of $R$ that maximize the k-width for a given volume? One candidate is a rectangle of dimensions $R_{1} \times \ldots \times R_{l} \times S \times \ldots \times S$, where $l \leq k-1$ and $S$ is between $R_{l}$ and $R_{l+1}$. We call this set $U_{0}$. The volume $V\left(U_{0}\right)$ is equal to $R_{1} \ldots R_{l} S^{n-l}$ and the k-width $W_{k}\left(U_{0}\right)$ is approximately $R_{1} \ldots R_{l} S^{k-l}$. Solving for $S$ in terms of $V\left(U_{0}\right)$ and plugging in, we see that $W_{k}\left(U_{0}\right)$ is roughly equal to $\left(R_{1} \ldots R_{l}\right)^{(n-k) /(n-l)} V\left(U_{0}\right)^{(k-l) /(n-l)}$. It turns out that $U_{0}$ has roughly the largest k-width among all subsets of $R$ with its volume. We now prove that any subset $U$ of $R$ obeys the inequality $W_{k}(U)<C(n)\left(R_{1} \ldots R_{l}\right)^{(n-k) /(n-l)} V(U)^{(k-l) /(n-l)}$. This inequality becomes roughly an equality when $U=U_{0}$.

Proposition 2.2. If $U$ is an open set contained in $R$, then for each integer $l$ in the range $0 \leq l \leq k$, the following inequality holds.

$$
W_{k}(U)<C(n)\left(R_{1} \ldots R_{l}\right)^{(n-k) /(n-l)} V(U)^{(k-l) /(n-l)} .
$$

Proof. When $l=0$, this inequality reduces to the width-volume inequality. When $l=k$, this inequality says that the width of $\mathrm{U}$ is less than $C(n) R_{1} \ldots R_{k}$. Since $U$ is a subset of $\mathrm{R}$, the width of $U$ is at most the width of $\mathrm{R}$, and this inequality follows. Now we turn to the intermediate values of 1 . 
Let $\mathrm{f}$ be the function on the (n-l)-dimensional rectangle $R_{l+1} \times \ldots \times R_{n}$ with $f(y)$ equal to $\left(R_{1} \ldots R_{l}\right)^{-1}$ times the volume of $U \cap\left[0, R_{1}\right] \times \ldots \times\left[0, R_{l}\right] \times\{y\}$. In other words, if $U$ contains all of $\left[0, R_{1}\right] \times \ldots \times\left[0, R_{l}\right] \times\{y\}$, then $f(y)$ will be 1 , and if $U$ contains half of that region, $f(y)$ will be $1 / 2$.

The function $\mathrm{f}$ is compactly supported, and $0 \leq f \leq 1$. Applying the widthvolume inequality for functions to the function $\mathrm{f}$, we see that the $(\mathrm{k}-\mathrm{l})$-width of $\mathrm{f}$ is bounded by $C(n)\left(\int f\right)^{\frac{k-l}{n-l}}$. This expression is equal to $C(n)\left[\left(R_{1} \ldots R_{l}\right)^{-1} V(U)\right]^{\frac{k-l}{n-l}}$. According to the definition of (k-l)-width, there is a family $\mathrm{F}$ of $(\mathrm{k}-\mathrm{l})$-cycles sweeping out the support of $\mathrm{f}$, so that the integral of $\mathrm{f}$ over each cycle $F(p)$ is bounded by this expression. We define a family $F^{\prime}$ of k-cycles sweeping out R. The family $F^{\prime}$ has the same parameter space as $\mathrm{F}$, and we define $F^{\prime}(p)=F(p) \times\left[0, R_{1}\right] \times \ldots \times\left[0, R_{l}\right]$. The volume of $\mathrm{U}$ intersected with a cycle $F^{\prime}(p)$ is bounded by $\left(R_{1} \ldots R_{l}\right)$ times the integral of $\mathrm{f}$ over the corresponding cycle $F(p)$. Therefore, the k-width of $U$ is bounded by $C(n)\left(R_{1} \ldots R_{l}\right)^{\frac{n-k}{n-l}} V(U)^{\frac{k-l}{n-l}}$.

This proposition allows us to estimate how many disjoint wide sets can be packed into a rectangle. We define $P_{k, N}(U)$ to be the supremal $\mathrm{W}$ so that there exist $\mathrm{N}$ disjoint subsets $U_{i} \subset U$ each with k-width at least $\mathrm{W}$. The letter $\mathrm{P}$ stands for packing-width. For a rectangle $R$, we can estimate $P_{k, N}(R)$ up to a constant factor $\mathrm{C}(\mathrm{n})$. The formula is a little complicated, but the geometric meaning is that cutting a rectangle $R$ into rectangular grids gives roughly the optimal packings.

Proposition 2.3. Suppose $R$ is an n-dimensional rectangle with dimensions $R_{1} \leq$ $\ldots \leq R_{n}$. Then, up to a factor of $C(n), P_{k, N}(R)$ is equal to the infimum of the following expression over all integers $l$ in the range $0 \leq l \leq k$ :

$$
R_{1} \ldots R_{l}\left(R_{l+1} \ldots R_{n}\right)^{\frac{k-l}{n-l}} N^{-\frac{k-l}{n-l}} .
$$

Proof. First we prove that $P_{k, N}(R)$ is bounded above by each of the expressions in the proposition. Let $U_{i}$ be $\mathrm{N}$ disjoint subsets of R. One of them must have volume at most $R_{1} \ldots R_{n} / N$. Applying the width volume inequality for rectangles, we see that this set has k-width less than $C(n)\left(R_{1} \ldots R_{l}\right)^{\frac{n-k}{n-l}}\left(R_{1} \ldots R_{n} N^{-1}\right)^{\frac{k-l}{n-l}}$, for each 1 between 0 and k. Expanding this expression, we get $R_{1} \ldots R_{l}\left(R_{l+1} \ldots R_{n}\right)^{\frac{k-l}{n-l}} N^{-\frac{k-l}{n-l}}$. This is the inequality we wanted to prove.

It remains to show that the packing-width is at least as great as this expression. To do this we will use the packing formed by cutting $R$ along a rectangular grid. First we consider the case $N \leq\left(R_{n} \ldots R_{k+1}\right) / R_{k}^{n-k}$. In this case, we can find $\mathrm{N}$ disjoint subrectangles in $R$ each of dimension $R_{1} \times \ldots \times R_{k} \times R_{k} \times \ldots \times R_{k}$. Each of these rectangles has k-width roughly $R_{1} \ldots R_{k}$. Therefore, $P_{k, N}(R) \geq c(n) R_{1} \ldots R_{k}$. Since the k-width of $R$ is at most $R_{1} \ldots R_{k}$, it follows that $P_{k, N}(R) \leq R_{1} \ldots R_{k}$, and this lower bound is sharp up to a constant factor. Second we consider the main case that $N \geq\left(R_{n} \ldots R_{k+1}\right) / R_{k}^{n-k}$. In this case, we can find $\mathrm{N}$ disjoint parallel rectangles in $R$ each with dimensions $R_{1} \times \ldots \times R_{l} \times \mathrm{S} \times \ldots \times S$, for some number $S$ in the range $R_{l} \leq \mathrm{S} \leq R_{l+1}$, where $l<k$. Moreover, we can choose these rectangles so that they fill up a good portion of the total volume of $\mathrm{R}$. In other words, $N R_{1} \ldots R_{l} S^{n-l}>c(n) R_{1} \ldots R_{n}$. Solving for $\mathrm{S}$, we see that $S>$ $c(n)\left(R_{n} \ldots R_{l+1}\right)^{\frac{1}{n-l}} N^{-\frac{1}{n-l}}$. Now the k-width of each rectangle is $R_{1} \ldots R_{l} S^{k-l}$, so we conclude that $P_{k, N}(R)>c(n) R_{1} \ldots R_{l}\left(R_{l+1} \ldots R_{n}\right)^{\frac{k-l}{n-l}} N^{-\frac{k-l}{n-l}}$. This lower bound 
comes within a constant factor of one of the upper bounds we proved in the first paragraph. Therefore, we have determined $P_{k, N}(R)$ up to a factor $C(n)$.

\section{Estimates OF K-Dilation}

In this section, we will estimate the k-dilation of degree 1 maps between certain domains in Euclidean space, especially rectangles. The first estimate follows from our knowledge of the k-width of rectangles, and the more refined estimates follow from our knowledge of the packing widths of rectangles. We begin by reviewing the definition of k-dilation and some of its basic properties.

Recall that a piecewise smooth map $\mathrm{f}$ has k-dilation at most $\lambda$ if $\mathrm{f}$ maps each $\mathrm{k}$-dimensional submanifold of the domain with volume $\mathrm{V}$ to an image with volume at most $\lambda V$. The $\mathrm{k}$-dilation of $\mathrm{f}$ can also be expressed in terms of the derivative $d f$. When $k=1$, the 1-dilation of $\mathrm{f}$ is equal to its Lipshitz constant, which is equal to the supremum of $|d f|$. We now generalize this result to all values of $\mathrm{k}$.

If $\mathrm{f}$ maps $\mathrm{M}$ to $\mathrm{N}$, then $d f$ at a point $\mathrm{m}$ maps $T M_{m}$ to $T N_{f(m)}$. Taking the k-fold exterior power of this map gives a map $\Lambda^{k} d f_{m}$ from $\Lambda^{k} T M_{m}$ to $\Lambda^{k} T N_{f(m)}$. By $\left|\Lambda^{k} d f_{m}\right|$, we denote the operator norm of this linear map. In other words, this norm is the maximum over all unit k-vectors $\mathrm{v}$ in $\Lambda^{k} T M_{m}$ of the norm $\left|\Lambda^{k} d f_{m}(v)\right|$.

Proposition 3.1. The $k$-dilation of a piecewise smooth map $f$ is equal to the supremum of $\left|\Lambda_{k} d f_{m}\right|$ as $m$ varies over $M$.

Proof. If the original derivative $d f_{m}$ has singular values $0 \leq s_{1} \leq \ldots \leq s_{n}$, corresponding to singular vectors $v_{1}, \ldots, v_{n}$, then the singular values of $\Lambda^{k} d f$ are given by all products of $\mathrm{k}$ distinct numbers $s_{i}$, and the singular $\mathrm{k}$-vectors are given by the wedge products of the corresponding vectors $v_{i}$. Therefore, it follows that the norm $\left|\Lambda^{k} d f_{m}\right|$ is equal to $s_{n-k+1} \ldots s_{n}$. Moreover, this singular value corresponds to a simple k-vector $v_{n-k+1} \wedge \ldots \wedge v_{n}$. Taking a small disk near $\mathrm{m}$ in the plane spanned by $v_{n-k+1}, \ldots, v_{n}$, we see that the k-dilation of $\mathrm{f}$ is at least $\left|\Lambda^{k} d f_{m}\right|$. On the other hand, the linear map $d f_{m}$ stretches the volume of each k-dimensional disk by at most $\left|\Lambda^{k} d f_{m}\right|$. By a standard calculus argument, the k-dilation of $\mathrm{f}$ is at most $\sup _{m}\left|\Lambda^{k} d f_{m}\right|$.

Formulating the k-dilation in terms of the singular values of the derivative allows us to show that the k-dilation controls the $(k+i)$-dilation for all $i>0$.

Proposition 3.2. Let $f$ be a piecewise smooth map with $k$-dilation at most $\lambda$. Then for each $i>0$, the $(k+i)$-dilation of $f$ is at most $\lambda^{\frac{k+i}{k}}$.

Proof. In the proof of the last proposition, we showed that the k-dilation of $\mathrm{f}$ is equal to the supremum of $s_{n-k+1} \ldots s_{n}$. Similarly, the $(k+i)$ dilation is equal to the supremum of $s_{n-k-i+1} \ldots s_{n}$. This expression is bounded by $s_{n-k+1}^{i} s_{n-k+1} \ldots s_{n}$. Since the k-dilation of $\mathrm{f}$ is at most $\lambda, s_{n-k+1} \leq \lambda^{1 / k}$. Plugging this bound into the last expression, we see that the $(k+i)$-dilation of $\mathrm{f}$ is at most $\lambda^{\frac{k+i}{k}}$.

We now turn to the main problem of this section. Let $U$ and $V$ be connected bounded open sets in $\mathbb{R}^{n}$ with piecewise smooth boundaries. Let $D_{k}(U, V)$ denote the infimal k-dilation of a degree 1 map from the pair $(U, \partial U)$ to the pair $(V, \partial V)$. How can we estimate $D_{k}(U, V)$ ?

To give some context, we first consider the more familiar cases when $\mathrm{k}$ is equal to $\mathrm{n}$ or to 1 . If $k=n$, we can get sharp estimates by using Moser's theorem for inducing 
differential forms. Suppose that $U$ and $V$ are diffeomorphic. Let $d v o l_{U}$ denote the volume form on $U$ and $d v o l_{V}$ denote the volume form on $V$. Moser proved in [10] that there is a diffeomorphism $\phi$ from $U$ to $V$ so that $\phi^{*} d v o l_{V}=\mu d v o l_{U}$, where $\mu$ is the ratio $\operatorname{Volume}(V) / \operatorname{Volume}(U)$. This diffeomorphism has n-dilation $\mu$. Since a degree 1 map is surjective, any degree 1 map from $U$ to $V$ must have n-dilation at least the ratio $\operatorname{Volume}(V) / \operatorname{Volume}(U)$. This result is very satisfactory, but it has no analogue for $k<n$. Next we consider the case $k=1$. For complicated domains $U$ and $V$, our problem may be difficult even for $k=1$. The distinguishing feature of $k=1$ is that we have a brute force approach which is not available for higher values of $\mathrm{k}$. If we fix bounded open sets $U$ and $V$, then the set of maps from $(U, \partial U)$ to $(V, \partial V)$ with 1-dilation at most $\lambda$ is compact in $C^{0}$. Therefore, at least in theory, one can systematically search this class of maps for maps of degree 1. This approach can be carried out on a computer if $U$ and $V$ are polyhedra, and it would give an estimate of $D_{1}(U, V)$ to arbitrary accuracy, although it would be extremely slow. By constrast, the set of maps with 2-dilation at most $\lambda$ is not compact in $C^{0}$, so that even with unlimited computing time I don't know how to systematically estimate $D_{2}(U, V)$ up to a factor of $10^{100}$.

We will focus on the special case of maps from a rectangle $R$ to a rectangle $S$. Even in this special case, the problem is much harder than I initially expected. We use the convention that the rectangle $R$ has dimensions $R_{1} \leq \ldots \leq R_{n}$ and the rectangle $S$ has dimensions $S_{1} \leq \ldots \leq S_{n}$. To make the algebra simpler, we let $Q_{i}$ denote the quotient $S_{i} / R_{i}$. We now prove some lower bounds for k-dilation.

Proposition 3.3. Suppose that $U$ is a subset of $R$. Then $D_{k}(U, S)$ is at least $c(n) Q_{1} \ldots Q_{k}$.

Proof. Since $U$ is a subset of $\mathrm{R}, W_{k}(R) \geq W_{k}(U)$. Now if there is a degree nonzero map from $U$ to $S$ with k-dilation $\lambda$, then $\lambda W_{k}(U) \geq W_{k}(S)$. Therefore, $\lambda \geq W_{k}(S) / W_{k}(R)$. But according to Proposition $1.2, W_{k}(S)$ is at least $c(n) S_{1} \ldots S_{k}$, and $W_{k}(R)$ is at most $R_{1} \ldots R_{k}$. Therefore, $\lambda$ is at least $c(n) Q_{1} \ldots Q_{k}$.

We can get more complicated bounds by considering the packing-widths of $R$ and $S$.

Proposition 3.4. Suppose that $U$ is a subset of $R$. Then, for each integer $l$ from 0 to $k, D_{k}(U, S)$ is at least $c(n) Q_{1} \ldots Q_{l}\left(Q_{l+1} \ldots Q_{n}\right)^{\frac{k-l}{n-l}}$.

Proof. Since $U$ is a subset of $R$, we have $P_{k, N}(R) \geq P_{k, N}(U)$ for every k and N. If there is a map $f$ of non-zero degree from $U$ to $S$ with k-dilation $\lambda$, then $\lambda P_{k, N}(U) \geq P_{k, N}(S)$. To see this, let $S_{i}$ be $\mathrm{N}$ disjoint subsets of S, each with width at least $P_{k, N}(S)-\epsilon$. Then let $U_{i}$ be the inverse image $F^{-1}\left(S_{i}\right)$. The map $f$ restricts to a degree non-zero map from $\left(U_{i}, \partial U_{i}\right)$ to $\left(S_{i}, \partial S_{i}\right)$. Therefore the k-width of $U_{i}$ is at least $\lambda^{-1}\left(P_{k, N}(S)-\epsilon\right)$. Since the sets $U_{i}$ are disjoint, $\lambda P_{k, N}(U) \geq P_{k, N}(S)$. This estimate gives us a lower bound $\lambda \geq P_{k, N}(S) / P_{k, N}(R)$, for every natural number N.

The value of $P_{k, N}(R)$ is estimated in Proposition 2.3. Up to a constant factor $\mathrm{C}(\mathrm{n})$, it is equal to the infimum of $R_{1} \ldots R_{l}\left(R_{l+1} \ldots R_{n}\right)^{\frac{k-l}{n-l}} N^{-\frac{k-l}{n-l}}$, where 1 lies in the range $0 \leq l \leq k$. In particular, we can consider the case that $N=S_{n} \ldots S_{l+1} / S_{l}^{n-l}$. Since there are roughly $\mathrm{N}$ disjoint rectangles in $S$ with dimensions $S_{1} \times \ldots \times S_{l} \times$ $S_{l} \times \ldots \times S_{l}$, the packing-width $P_{k, N}(S)$ is at least $c(n) S_{1} \ldots S_{l} S_{l}^{k-l}$. On the other 
hand, $P_{k, N}(R)$ is at most $C(n) R_{1} \ldots R_{l}\left(R_{l+1} \ldots R_{n}\right)^{\frac{k-l}{n-l}}\left[S_{n} \ldots S_{l+1} / S_{l}^{n-l}\right]^{-\frac{k-l}{n-l}}$. Therefore, $P_{k, N}(S) / P_{k, N}(R)$ is at least $c(n) Q_{1} \ldots Q_{l}\left(Q_{l+1} \ldots Q_{n}\right)^{\frac{k-l}{n-l}}$.

This finishes the proof of the proposition. The reader can check that the supremum over $\mathrm{N}$ of the quotient $P_{k, N}(S) / P_{k, N}(R)$ is approximately equal to the maximum of $Q_{1} \ldots Q_{l}\left(Q_{l+1} \ldots Q_{n}\right)^{\frac{k-l}{n-l}}$ for 1 in the range $0 \leq l \leq k$. Therefore, the packingwidth does not give any further lower bounds for $\bar{D}_{k}(\bar{R}, S)$.

The second theorem of this paper is an estimate for $D_{n-1}(R, S)$.

Theorem 2. Let $R$ and $S$ be $n$-dimensional rectangles. Suppose that $R$ has dimensions $R_{1} \leq \ldots \leq R_{n}$ and that $S$ has dimensions $S_{1} \leq \ldots \leq S_{n}$. Up to a constant factor $C(n), D_{n-1}(R, S)$ is equal to the supremum of the following quantities:

$$
\begin{gathered}
Q_{1} \ldots Q_{l}\left(Q_{l+1} \ldots Q_{n}\right)^{\frac{n-l-1}{n-l}} \\
Q_{2} \ldots Q_{n} .
\end{gathered}
$$

In equation (1), the number $l$ is allowed to take any value in the range $1 \leq l \leq n-1$.

For example, if $\mathrm{n}$ is 3 , then $D_{2}(R, S)$ is roughly the supremum of $Q_{1} Q_{2}^{1 / 2} Q_{3}^{1 / 2}$, $Q_{1} Q_{2}$, and $Q_{2} Q_{3}$.

We have already proven the lower bounds in equation 1 . They are exactly the lower bounds in Proposition 3.4 in case $k=n-1$.

The lower bound in equation 2 is simple. Suppose that $f$ is a degree 1 map from $R$ to $S$ with $(n-1)$-dilation $\lambda$. The map f restricts to a degree 1 map from the boundary of $R$ to the boundary of $\mathrm{S}$. Since this map must be surjective, it follows that $\lambda \operatorname{Volume}(\partial R) \geq \operatorname{Volume}(\partial S)$. But the volume of the boundary of $\mathrm{R}$ is at most $2 n R_{2} \ldots R_{n}$, and the volume of the boundary of $\mathrm{S}$ is at least $2 S_{2} \ldots S_{n}$. Therefore, $\lambda$ is at least $(1 / n) Q_{2} \ldots Q_{n}$. This finishes the proof of the lower bounds on $D_{n-1}(R, S)$.

In the next section, we will construct degree 1 maps showing that these lower bounds are sharp up to a constant factor.

\section{Maps With SMALL $(n-1)$-Dilation}

In this section, we construct a degree 1 map between rectangles with surprisingly small k-dilation. After the construction, we check that the k-dilation of this map can be smaller than the k-dilation of any linear diffeomorphism by an arbitrarily large factor. Then we will finish the proof of Theorem 2, determining $D_{n-1}(R, S)$ up to a constant factor.

Construction 2. (The snake map) Let $R$ and $S$ be $n$-dimensional rectangles. Suppose that $n \geq 3$ and that $k$ lies in the range $2 \leq k \leq n-1$. Suppose that $R_{i}=S_{i}$ for $i \leq n-k$. Suppose that $R_{n-k+1} \ldots R_{n-k+b} \geq S_{n-k+1} \ldots S_{n-k+b}$ for every $b$ in the range $1 \leq b \leq k$. Then there is a degree 1 map from $R$ to $S$ with $k$-dilation less than $C(n)$.

Proof. We write $R$ as the product $R^{\prime} \times R^{\prime \prime}$, where $R^{\prime}=\left[0, R_{1}\right] \times \ldots \times\left[0, R_{n-k}\right]$ and $R^{\prime \prime}=\left[0, R_{n-k+1}\right] \times \ldots \times\left[0, R_{n}\right]$. Similarly, we write $S=S^{\prime} \times S^{\prime \prime}$. By assumption $R^{\prime}$ is congruent to $S^{\prime}$.

Because of the inequalities $R_{n-k+1} \ldots R_{n-k+b} \geq S_{n-k+1} \ldots S_{n-k+b}$, there is a smooth bilipschitz embedding of $S^{\prime \prime}$ into $R^{\prime \prime}$ with bilipschitz constant at most $C(n)$. 
We will need a little bit of room later, so we let $I$ be a smooth bilipschitz embedding of $3 S^{\prime \prime}$ into $R^{\prime \prime}$, with quasi-isometric constant $C(n)$. (By $3 S^{\prime \prime}$, we mean the rectangle $S^{\prime \prime}$ dilated by a factor of 3 around its center.) We let A be the image of $\mathrm{I}$ in $R^{\prime \prime}$. (This set A corresponds to the set $\mathrm{A}$ in the description of the snake map before this proof.) Let $\mathrm{H}$ be a smooth function on $3 S^{\prime \prime}$ which is equal to 1 on the central $S^{\prime \prime}$ and is equal to zero on a neighborhood of the boundary of $S^{\prime \prime}$. We can choose $\mathrm{H}$ with Lipschitz constant as close as we like to $S_{n-k+1}^{-1}$.

The function $H \circ I^{-1}$ is defined on the image of $I$ in $R^{\prime \prime}$, and it is equal to zero on the boundary of this image. We extend this function to all of $R^{\prime \prime}$ by setting it equal to zero on the complement of the image of I. We call the resulting function $\bar{H}$. We denote a point in $R$ by $\left(x^{\prime}, x^{\prime \prime}\right)$, where $x^{\prime}$ lies in $R^{\prime}$ and $x^{\prime \prime}$ lies in $R^{\prime \prime}$. We define $\Phi_{1}\left(x^{\prime}, x^{\prime \prime}\right)=\left(\bar{H}\left(x^{\prime \prime}\right) x^{\prime}, x^{\prime \prime}\right)$. If we differentiate $\Phi_{1}$, we find that the norm of the derivative is bounded by the sum $\sup \left|H\left(x^{\prime \prime}\right)\right|+\sup \left|x^{\prime}\right| \sup |\nabla \bar{H}|$. The first of these expressions is bounded by 1 , and the second by $C(n) R_{n-k} / S_{n-k+1}$. Because of our assumptions about the dimensions of $R$ and $\mathrm{S}$, we have $R_{n-k}=S_{n-k} \leq S_{n-k+1}$, and so the map $\Phi_{1}$ has Lipschitz constant less than C. The image $\Phi_{1}(R)$ is contained in $R^{\prime} \times A \cup\{0\} \times R^{\prime \prime}$. We call this set Q.

The next step of our construction is to retract the region $\mathrm{Q}$ onto $R^{\prime} \times A$. To do this, we first pick a retraction $\phi_{2}$ from $R^{\prime \prime}$ to the image of I. We choose $\phi_{2}$ so that it maps the complement of A onto the boundary of A. We also assume that $\phi_{2}$ is piecewise smooth. Next, we define $\Phi_{2}\left(x^{\prime}, x^{\prime \prime}\right)=\left(x^{\prime}, \phi_{2}\left(x^{\prime \prime}\right)\right)$.

The map $\Phi_{2}$ has large k-dilation on $\mathrm{R}$, but its restriction to $\mathrm{Q}$ has k-dilation 1 . On the intersection of $\mathrm{Q}$ with the region $R^{\prime} \times A, \Phi_{2}$ is the identity, and so it has $\mathrm{k}$-dilation 1. The complement of this region in $\mathrm{Q}$ is given by the conditions $x^{\prime}=0$ and $x^{\prime \prime} \in A^{c}$, where $A^{c}$ denotes the complement of $\mathrm{A}$ in $R^{\prime \prime}$. The map $\Phi_{2}$ takes this $k$-dimensional region into the $(k-1)$-dimension region given by the conditions $x^{\prime}=0$, and $x^{\prime \prime} \in \partial A$. Therefore, $\Phi_{2}$ has k-dilation zero on the second part of $\mathrm{Q}$. All together, the map $\Phi_{2}$ has k-dilation 1 .

Next, we define a map $\Phi_{3}$ from the region $R^{\prime} \times A$ to $S^{\prime} \times 3 S^{\prime \prime}$. This map is defined by $\Phi_{3}\left(x^{\prime}, x^{\prime \prime}\right)=\left(x^{\prime}, I^{-1}\left(x^{\prime \prime}\right)\right)$. It has Lipschitz constant at most $C(n)$. The composition $\Phi_{3} \circ \Phi_{2} \circ \Phi_{1}$ is a map of $R$ into $S^{\prime} \times 3 S^{\prime \prime}$, with k-dilation less than $C(n)$. The rectangle $S^{\prime} \times 3 S^{\prime \prime}$ contains the rectangle $S=S^{\prime} \times S^{\prime \prime}$. Since $S$ is convex, there is a retraction $\Phi_{4}$ from $S^{\prime} \times 3 S^{\prime \prime}$ to $S$ with Lipschitz constant 1 .

The composition $\Phi_{4} \circ \Phi_{3} \circ \Phi_{2} \circ \Phi_{1}$ is a degree 1 map from $(R, \partial R)$ to $(S, \partial S)$ with k-dilation less than $C(n)$.

Remark: With a little more work, it is possible to construct a PL isomorphism from $R$ to $S$ with k-dilation less than $C(n)$.

We now give an example to show that the snake map badly outperforms the linear map for some rectangles. Let $\operatorname{Lin}_{k}(R, S)$ denote the smallest k-dilation of any linear diffeomorphism from $R$ to $S$.

Proposition 4.1. For each $n \geq 3$ and each $k$ in the range $2 \leq k \leq n-1$, there are $n$-dimensional rectangles $R$ and $S$ which make the ratio $\operatorname{Lin}_{k}(R, S) / D_{k}(R, S)$ arbitrarily large.

Proof. Let $R$ be the rectangle with dimensions $R_{1}=\ldots=R_{n-2}=\epsilon$, and $R_{n-1}=$ $R_{n}=1$. Let $S$ be the rectangle with dimensions $S_{1}=\ldots=S_{n-1}=\epsilon$ and $S_{n}=\epsilon^{-1}$. Construction 2 gives a degree 1 map from $\mathrm{R}$ to $S$ with 2-dilation at most $C(n)$. Next we give a lower bound for $\operatorname{Lin}_{n-1}(R, S)$. Any linear diffeomorphism from 
$R$ to $\mathrm{S}$ takes each hyperface of $R$ onto a hyperface of $\mathrm{S}$. The rectangle $\mathrm{R}$ has 4 hyperfaces with volume $\epsilon^{n-2}$. On the other hand, the rectangle $S$ has $2(n-1)$ hyperfaces with volume $\epsilon^{n-3}$ and only 2 hyperfaces with volume $\epsilon^{n-2}$. Any linear diffeomorphism from $R$ onto $S$ must take a face of $R$ with volume $\epsilon^{n-2}$ onto a face of $\mathrm{S}$ with volume $\epsilon^{n-3}$. Therefore, it must have (n-1)-dilation at least $\epsilon^{-1}$, and we conclude that $\operatorname{Lin}_{n-1}(R, S) \geq \epsilon^{-1}$.

According to Proposition 3.2, a map with k-dilation $\lambda$ has $(k+i)$-dilation at most $\lambda^{\frac{k+i}{k}}$. Therefore, $D_{k}(R, S)$ is less than $C(n)$ for all $k \geq 2$. By the same argument, $\operatorname{Lin}_{k}(R, S)$ is at least $\epsilon^{-\frac{k}{n-1}}$ for all $k \leq n-1$. Combining these estimates, we see that $\operatorname{Lin}_{k}(R, S) / D_{k}(R, S)$ may be arbitrarily large for all $\mathrm{k}$ in the range $2 \leq k \leq n-1$.

Using the snake map, we now finish the proof of Theorem 2 .

Proof. By composing snake maps and linear maps, we will construct enough degree 1 maps to prove the theorem. We begin with the case $n=3$.

By scaling the rectangle $\mathrm{S}$, we can assume that the lower bound for $D_{2}(R, S)$ given in Theorem 2 is equal to 1 . In other words, we suppose $R_{1} R_{2}>S_{1} S_{2}$, $R_{1}^{2} R_{2} R_{3}>S_{1}^{2} S_{2} S_{3}$, and $R_{2} R_{3}>S_{2} S_{3}$. Under these assumptions, we need to construct a degree 1 map from $R$ to $S$ with 2-dilation less than C. We do so in three cases.

If $R_{1}<S_{1}$, then we define a 2-contracting linear diffeomorphism from $R$ to T, with $T_{1}=S_{1}, T_{2}=R_{2} R_{1} / S_{1}$, and $T_{3}=R_{3} R_{1} / S_{1}$. (The length $T_{2}$ is indeed bigger than $T_{1}$ because $R_{1} R_{2}>S_{1} S_{2}$.) Using the first two equations in the list above, we see that $T_{2}>S_{2}$ and $T_{2} T_{3}>S_{2} S_{3}$. Therefore, there is a snake map from T to $S$ with 2-dilation less than $\mathrm{C}$

If $R_{1} \geq S_{1}$ but $R_{2}<S_{2}$, then we define a 2-contracting linear diffeomorphism from $R$ to $\mathrm{T}$, with $T_{1}=R_{1} R_{2} / S_{2}, T_{2}=S_{2}$, and $T_{3}=R_{3} R_{2} / S_{2}$. (The length $T_{3}$ is indeed bigger than $T_{2}$ because $R_{2} R_{3}>S_{2} S_{3}$.) Since $R_{1} R_{2}>S_{1} S_{2}, T_{1}>S_{1}$. Since $R_{2} R_{3}>S_{2} S_{3}, T_{3}>S_{3}$. Therefore, there is a 1-contracting linear diffeomorphism from $\mathrm{T}$ to $\mathrm{S}$.

If $R_{1} \geq S_{1}$ and $R_{2} \geq S_{2}$, since we have assumed that $R_{2} R_{3} \geq S_{2} S_{3}$, there is a snake map from $R$ to $S$ with 2-dilation less than C.

We now turn to the case of higher dimensions. As in the three-dimensional case, we can scale $S$ so that the lower bound on $D_{n-1}(R, S)$ is equal to 1 . In other words, we can assume that the rectangles $R$ and $S$ obey the following list of inequalities denoted $(*)$ :

$$
\begin{gathered}
R_{1} \ldots R_{l}\left(R_{l+1} \ldots R_{n}\right)^{\frac{n-l-1}{n-l}}>S_{1} \ldots S_{l}\left(S_{l+1} \ldots S_{n}\right)^{\frac{n-l-1}{n-l}} . \\
R_{2} \ldots R_{n} \geq S_{2} \ldots S_{n} .
\end{gathered}
$$

Equation $(* 1)$ holds for every integer $l$ in the range $1 \leq l \leq n-1$.

Assuming (*), we have to construct a degree 1 map from $R$ to $S$ with $(n-$ 1)-dilation at most $C(n)$. The maps we will construct will have the following structure. First, there will be a snake map from $R$ to an intermediate rectangle $T$, with (n-1)-dilation at most $C(n)$. Then there will be an (n-1)-contracting linear diffeomorphism from $T$ to $S$. Choosing the rectangle $T$ and constructing the two maps is very tedious, but it requires only elementary algebra. 
We begin with the special case $R_{1}=S_{1}$. Because of the messy algebra, from now on we use $C$ to denote a constant that depends only on $\mathrm{n}$.

Lemma 4.1. Suppose that $R$ and $S$ are $n$-dimensional rectangles obeying (*). Also suppose that $R_{1}=S_{1}$, and suppose that Theorem 2 holds for rectangles of dimension $n$-1. Then there is a degree 1 map from $R$ to $S$ with (n-1)-dilation at most $C$.

Proof. Since $R_{1}=S_{1}$, the inequalities in $(*)$ imply that for each 1 in the range $1 \leq l \leq n-1, R_{2} \ldots R_{l}\left(R_{l+1} \ldots R_{n}\right)^{\frac{n-l-1}{n-l}} \geq S_{2} \ldots S_{l}\left(S_{l+1} \ldots S_{n}\right)^{\frac{n-l-1}{n-l}}$.

We are going to prove something a little more general in order to do an inductive argument. For each $\mathrm{p}$ in the range $1 \leq p \leq n-1$, let $C(p)$ denote the following list of conditions.

1. $R_{1}=S_{1}$.

2. $R_{2} \ldots R_{a} \geq S_{2} \ldots S_{a}$ for a in the range $2 \leq a \leq p$,

3. $R_{2} \ldots R_{l}\left(R_{l+1} \ldots R_{n}\right)^{\frac{n-l-1}{n-l}} \geq S_{2} \ldots S_{l}\left(S_{l+1} \ldots S_{n}\right)^{\frac{n-l-1}{n-l}}$ for 1 in the range $p \leq l \leq$ $n-1$, and

4. $R_{2} \ldots R_{n} \geq S_{2} \ldots S_{n}$.

Our hypotheses are exactly $C(1)$. We are going to prove that for every p, $C(p)$ implies that there is a degree 1 map from $\mathrm{R}$ to $S$ with $(n-1)$-dilation at most C. The point of introducing all of these new conditions is that we can make an inductive argument, starting with $C(n-1)$ and working our way down to $C(1)$.

To anchor the induction we prove that $C(n-1)$ implies that there is a degree 1 map from $R$ to $S$ with $(n-1)$-dilation at most C. The condition $C(n-1)$ says exactly that $R_{1}=S_{1}$, and that $R_{2} \ldots R_{a} \geq S_{2} \ldots S_{a}$ for every a. Under these conditions, our construction gives a snake map from $R$ to $S$ with $(n-1)$-dilation at most C.

We now turn to the inductive step. We may assume that $C(q)$ implies a good map for every $q$ greater than $\mathrm{p}$, and we have to prove that $C(p)$ implies the existence of a good map.

Suppose that $R$ and $S$ satisfy $C(p)$. Let b be the smallest number for which $R_{2} \ldots R_{b}<S_{2} \ldots S_{b}$. If there is no such b, then $R$ and $S$ actually satisfy $C(n-1)$, and so there is a snake map from $R$ to $S$ with (n-1)-dilation at most $C$. Because of condition 4 we know that $\mathrm{b}$ is not equal to $\mathrm{n}$, and because of the condition 3 with $l=n-1$, we know that $\mathrm{b}$ is not equal to $\mathrm{n}-1$. On the other hand, $b$ must be greater than $p$. Therefore, b lies in the range $p<b \leq n-2$.

We begin with case $b=2$, which is a bit easier than the general case. If $b=2$, then we must have had $p=1$. There is an (n-1)-contracting linear diffeomorphism from $T$ to $S$, where $T_{1}=S_{1}, T_{2}=R_{2}$, and $T_{i}=S_{i}\left(S_{2} / R_{2}\right)^{1 /(n-3)}$ for all $i \geq 3$. (The length $T_{2}$ is at least $T_{1}$ because $T_{1}=S_{1}=R_{1} \leq R_{2}=T_{2}$.) We check that $R$ and $T$ obey $C(2)$. Condition 1 follows because $R_{1}=S_{1}=T_{1}$. Condition 2 follows because $R_{2}=T_{2}$. A computation shows that for $l$ in the range $2 \leq l \leq n-1$, $T_{2} \ldots T_{l}\left(T_{l+1} \ldots T_{n}\right)^{\frac{n-l-1}{n-l}}=S_{2} \ldots S_{l}\left(S_{l+1} \ldots S_{n}\right)^{\frac{n-l-1}{n-l}}$. Therefore, condition 3 of $C(2)$ holds. Finally, condition 4 follows from condition 3 for $\mathrm{l}=2$ along with the equality $T_{2}=R_{2}$. Since $p=1$, our inductive hypothesis is that $C(2)$ implies the existence of a good map. Therefore we may conclude there is a good map from $R$ to $T$. Composing with the linear map from $T$ to $S$ gives a good map from $R$ to $S$.

Now we deal with the more general case that $b>2$. In this case, we apply an (n-1)-expanding linear transformation to $S$ that leaves $S_{1}$ through $S_{b-1}$ invariant, decreases $S_{b}$ by some factor $\lambda$, and increases $S_{b+1}$ through $S_{n}$ by a factor $\lambda^{\frac{1}{n-b-1}}$. (At this stage, we use the fact that $b<n-1$.) We choose $\lambda$ so that the image 
rectangle $S^{\prime}$ has either $R_{2} \ldots R_{b}=S_{2}^{\prime} \ldots S_{b}^{\prime}$, or $S_{b-1}^{\prime}=S_{b}^{\prime}$, whichever requires a smaller value of $\lambda$. This choice of $\lambda$ ensures that the dimensions of $S^{\prime}$ are still labelled in increasing order. If $R_{2} \ldots R_{b}=S_{2}^{\prime} \ldots S_{b}^{\prime}$, then we can stop, but if $S_{b-1}^{\prime}=S_{b}^{\prime}$, we have to proceed with another (n-1)-expanding linear transformation. In this case, we then apply an (n-1)-expanding linear transformation to $S^{\prime}$ that leaves $S_{1}^{\prime}$ through $S_{b-2}^{\prime}$ invariant, decreases $S_{b-1}^{\prime}$ and $S_{b}^{\prime}$ equally, and increases all the other directions equally. We choose the factor of stretching so that the image rectangle $S^{\prime \prime}$ has either $R_{2} \ldots R_{b}=S_{2}^{\prime \prime} \ldots S_{b}^{\prime \prime}$ or $S_{b-2}^{\prime \prime}=S_{b-1}^{\prime \prime}=S_{b}^{\prime \prime}$. In the latter case, we then apply a linear transformation that decreases $S_{b-2}^{\prime \prime}, S_{b-1}^{\prime \prime}$, and $S_{b}^{\prime \prime}$, and so on. Because $R_{2}>S_{2}$, this process terminates. We call the final rectangle in this chain of (n-1)-expanding diffeomorphisms $T$.

At the end of the process, we have an equality $R_{2} \ldots R_{b}=T_{2} \ldots T_{b}$. We check that the rectangles $R$ and $T$ obey the condition $C(b)$. Condition 1 follows because $R_{1}=S_{1}=T_{1}$. Condition 2 follows because $R_{2} \ldots R_{b}=T_{2} \ldots T_{b}$, and because for each $a$ less than $b, T_{2} \ldots T_{a} \leq S_{2} \ldots S_{a} \leq R_{2} \ldots R_{a}$. A calculation shows that $T_{2} \ldots T_{l}\left(T_{l+1} \ldots T_{n}\right)^{\frac{n-l-1}{n-l}}=S_{2} \ldots S_{l}\left(S_{l+1} \ldots S_{n}\right)^{\frac{n-l-1}{n-l}}$ for $l \geq b$. Therefore, condition 3 of $C(b)$ holds for $R$ and $T$. Finally condition 4 follows from the case $l=b$ of condition 3 along with the equality $R_{2} \ldots R_{b}=T_{2} \ldots T_{b}$. Since $b$ is greater than $p$, our inductive hypothesis tells us that there is a degree 1 map from $R$ to $T$ with (n-1)dilation at most $C$. Since there is an (n-1)-contracting linear diffeomorphism from $T$ to $S$, we can compose them to get a degree 1 map from $R$ to $S$ with $(n-1)$-dilation at most $C$.

This argument proves the inductive step and hence the lemma.

With the help of the lemma, we can now prove Theorem 2 for all pairs of rectangles $R$ and $S$. We assume that $R$ and $S$ obey $(*)$, and we need to construct a degree 1 map from $R$ to $S$ with (n-1)-dilation at most C.

If $R_{1}<S_{1}$, then we proceed as follows. There is an (n-1)-contracting linear map from $T$ to $S$, where $T$ has dimensions $T_{1}=R_{1}$, and $T_{i}=S_{i}\left(S_{1} / R_{1}\right)^{\frac{1}{n-2}}$ for all other i. A calculation shows that $T_{1} \ldots T_{l}\left(T_{l+1} \ldots T_{n}\right)^{\frac{n-l-1}{n-l}}=S_{1} \ldots S_{l}\left(S_{l+1} \ldots S_{n}\right)^{\frac{n-l-1}{n-l}}$ for each 1 in the range $1 \leq l \leq n-1$. Since $R$ and $S$ obey (*), it follows that $R_{1} \ldots R_{l}\left(R_{l+1} \ldots R_{n}\right)^{\frac{n-l-1}{n-l}} \geq T_{1} \ldots T_{l}\left(T_{l+1} \ldots T_{n}\right)^{\frac{n-l-1}{n-l}}$ for each 1 in the range $1 \leq l \leq$ $n-1$. Since $R_{1}=T_{1}$, the last equation in the case $l=1$ implies that $R_{2} \ldots R_{n} \geq$ $T_{2} \ldots T_{n}$. In other words, $R$ and $T$ obey $(*)$. Since $R_{1}=T_{1}$, we can apply Lemma 4.1, which tells us that there is a degree 1 map from $R$ to $T$ with $(n-1)$-dilation less than C. Composing with the linear map from $T$ to $S$ gives a degree 1 map from $R$ to $S$ with $(n-1)$-dilation less than $\mathrm{C}$, which is what we wanted to prove.

If $R_{1}>S_{1}$, then we proceed as follows. We apply an (n-1)-expanding linear transformation to $S$ which decreases $S_{2}$ and increases all other directions of $S$ equally until either $S_{1}=R_{1}$ or $S_{1}=S_{2}$. In the second case, we apply an (n-1)expanding linear transformation to $S$ which decreases $S_{3}$ and increases all other directions of $S$ equally until either $S_{1}=R_{1}$ or $S_{1}=S_{2}=S_{3}$, and so on. We continue this process until either $S_{1}=R_{1}$ or $S_{1}=S_{n}<R_{1}$. In the latter case, there is a contracting linear diffeomorphism from $R$ to $S$.

In the former case, we call the final rectangle in this chain of (n-1)-expanding linear diffeomorphisms $T$. We have to check that $R$ and $T$ obey (*). Suppose that the last diffeomorphism was decreasing $S_{b+1}$ and increasing $S_{i}$ for all other i. We have $R_{1}=T_{1}=T_{2}=\ldots=T_{b}$. If $l$ is at least $b+1$, then a calculation 
shows that $T_{1} \ldots T_{l}\left(T_{l+1} \ldots T_{n}\right)^{\frac{n-l-1}{n-l}}=S_{1} \ldots S_{l}\left(S_{l+1} \ldots S_{n}\right)^{\frac{n-l-1}{n-l}}$. This equation implies that $R_{1} \ldots R_{l}\left(R_{l+1} \ldots R_{n}\right)^{\frac{n-l-1}{n-l}} \geq T_{1} \ldots T_{l}\left(T_{l+1} \ldots T_{n}\right)^{\frac{n-l-1}{n-l}}$ for $l \geq b+1$. Another short calculation shows that $T_{2} \ldots T_{n}=S_{2} \ldots S_{n}$. Therefore, $R_{2} \ldots R_{n} \geq T_{2} \ldots T_{n}$. Since $T_{1}=$ $T_{b}=R_{1}$, it follows that $R_{1} \ldots R_{l} \geq T_{1} . . T_{l}$ for all $l$ in the range $1 \leq l \leq b$. Since $R_{1}=$ $T_{1}$ and $R_{2} \ldots R_{n} \geq T_{2} \ldots T_{n}$, it follows that $R_{1} \ldots R_{n} \geq T_{1} \ldots T_{n}$. Combining the last two inequalities, it follows that $R_{1} \ldots R_{l}\left(R_{l+1} \ldots R_{n}\right)^{\frac{n-l-1}{n-l}} \geq T_{1} \ldots T_{l}\left(T_{l+1} \ldots T_{n}\right)^{\frac{n-l-1}{n-l}}$, for all $b$ in the range $1 \leq l \leq b$. Assembling all these inequalities, we see that $R$ and $T$ obey $(*)$.

Since $R_{1}=T_{1}$, we can apply Lemma 4.1 , which tells us that there is a degree 1 map from $R$ to $T$ with $(n-1)$-dilation less than C. Composing with the linear diffeomorphism from $T$ to $S$ gives a degree 1 map from $R$ to $S$ with $(n-1)$-dilation less than $\mathrm{C}$.

\section{Appendix: Dividing AREa And (N-1)-Width}

In this appendix, we briefly consider estimating the width of a Riemannian manifold $\left(M^{n}, g\right)$. The main result is that for each $n \geq 2$ a closed oriented Riemannian n-manifold with volume 1 may have arbitrarily large (n-1)-width. Therefore, the width-volume inequality does not extend to Riemannian manifolds.

Our results are based on isoperimetric inequalities. Let $\left(M^{n}, g\right)$ be a closed Riemannian manifold. We define the dividing area of $(M, g)$ to be the infimum of the volume of $\partial A$ as $A \subset M$ varies over all open sets with volume between $(1 / 4) \operatorname{Volume}(M)$ and $(3 / 4) \operatorname{Volume}(M)$. Any isoperimetric inequality on $(M, g)$ will lead to a lower bound for its dividing area.

Proposition 5.1. Let $(M, g)$ be a closed oriented Riemannian n-manifold with dividing area $A(M, g)$.

$$
W_{n-1}(M, g) \geq(1 / 2) A(M, g)
$$

Recall that the Cheeger isoperimetric constant $h(M, g)$ is equal to the supremum of $\operatorname{vol}(A) / \operatorname{vol}(\partial A)$ as A ranges over all open sets in $(M, g)$ with volume at most $(1 / 2) \operatorname{vol}(M)$. In [3], Brooks constructed examples of Riemannian manifolds with arbitrarily large volume and with Cheeger constant bounded below. We state Brooks's result as a theorem.

Theorem. (Brooks) For each $n \geq 2$, there is a sequence of closed oriented Riemannian $n$-manifolds $\left(M_{i}, g_{i}\right)$ with volume $V_{i}$ tending to infinity and $h\left(M_{i}, g_{i}\right)>1$.

The Riemannian manifolds $\left(M_{i}, g_{i}\right)$ are finite coverings of a fixed base manifold with an interesting fundamental group. The base manifold could be hyperbolic, or a higher-rank symmetric space. Because of the lower bound on the Cheeger constant, the dividing area of $\left(M_{i}, g_{i}\right)$ must be at least $(1 / 4) V_{i}$. According to Proposition 5.1, the (n-1)-width of $\left(M_{i}, g_{i}\right)$ is at least $(1 / 8) V_{i}$. Now we rescale $\left(M_{i}, g_{i}\right)$ so that it has volume 1 . The rescaled version has $(n-1)$-width at least $(1 / 8) V_{i}^{1 / n}$ which tends to infinity.

In Brooks's examples, the topology of $M_{i}$ is unbounded. There are other examples on the $n$-sphere for $n \geq 3$. These examples follow from the isoperimetric estimates of Burago and Ivanov, proven in 4]. A small modification of their construction gives the following theorem. 
Theorem. (Burago, Ivanov) For each $n \geq 3$ and each $\epsilon>0$, there is a metric $g$ on $S^{n}$ which obeys the following isoperimetric inequality. For any open set $A \subset S^{n}$ with volume between $(1 / 4) \operatorname{Volume}\left(S^{n}, g\right)$ and $(3 / 4) \operatorname{Volume}\left(S^{n}, g\right)$,

$$
\operatorname{Volume}(A)^{\frac{n-1}{n}}<\epsilon \operatorname{Volume}(\partial A) \text {. }
$$

Since this isoperimetric inequality is scale invariant, we can also scale the metrics so that they all have volume 1 . In this case, we have $(1 / 4) \leq \operatorname{Volume}(A)^{\frac{n-1}{n}} \leq$ $\epsilon \operatorname{Volume}(\partial A)$. Therefore, these metrics have dividing area at least $(1 / 4) \epsilon^{-1}$. By Proposition 5.1, we conclude that $W_{n-1}\left(S^{n}, g\right) \geq(1 / 8) \epsilon^{-1}$.

Now we turn to the proof of Proposition 5.1.

Proof. Let $\mathrm{F}$ be a family of (n-1)-cycles sweeping out $(M, g)$ with width almost $W_{n-1}(M, g)$. Without loss of generality we may assume that the parameter space of $\mathrm{F}$ is a circle. As in section 1, we perturb $\mathrm{F}$ to get a complex of cycles, where each 0 -simplex gets mapped to an (n-1)-cycle in $\mathrm{M}$ with mass at most $W_{n-1}(M, g)+\delta$ and each 1-simplex gets mapped to an n-chain with mass at most $\delta$.

Let $I$ be an interval of the circle consisting of a union of 1-simplices, and consider the union of the corresponding $\mathrm{n}$-chains, which we denote $C(I)$. We consider the image of $C(I)$, which is a set in $(M, g)$. If $I$ is a single 1 -simplex, this set has volume at most $\delta$. If $I$ is the whole circle, then this set is all of $(M, g)$. Since adding a 1 -simplex only slightly changes the volume of this set, we can find an interval $I$ so that the volume of the image of $C(I)$ is close to $(1 / 2) \operatorname{Volume}(M, g)$. Therefore, the volume of the boundary of this image is at least the dividing area of $(M, g)$. Now let $v_{1}$ and $v_{2}$ be the boundary vertices of I. The boundary of the image of $C(I)$ is contained in the union $C\left(v_{1}\right) \cup C\left(v_{2}\right)$. Therefore, one of these two cycles must have mass at least $(1 / 2) A(M, g)$. Taking $\delta \rightarrow 0$ finishes the proof.

\section{Appendix: Falconer's estimate for the linear K-Width}

In [5], Falconer proved the following theorem, which we have reformulated in our language.

Theorem. (Falconer) Let $U$ be a bounded open set in $\mathbb{R}^{n}$. Suppose that $k>n / 2$. Then there is a family of parallel $k$-planes, each interesecting $U$ in a region of $k$-volume at most $C(n) \operatorname{Volume}(U)^{k / n}$.

The proof is based on Fourier analysis. We give a sketch in the simplest case: $k=2, n=3$. By a scaling argument, we can assume that the volume of $U$ is 1 , and we let $f$ denote the characteristic function of $U$. Then we consider the Fourier transform of $f$. Because $\|f\|_{1}=1$, we have $\|\hat{f}\|_{\infty} \leq 1$. Because $\|f\|_{2}=1$, the Plancherel theoerem tells us that $\|\hat{f}\|_{2}=1$. We write this last equation in polar coordinates.

$$
\int_{S^{2}} \int_{0}^{\infty}|\hat{f}(\theta, r)|^{2} r^{2} d r d \theta=1
$$

It's convenient to expand the polar coordinates so that the radius takes values on the whole real line by identifying $(\theta,-r)$ with $(-\theta, r)$.

$$
\int_{S^{2}} \int_{-\infty}^{\infty}|\hat{f}(\theta, r)|^{2} r^{2} d r d \theta=2
$$


Since the unit sphere has area $4 \pi$, we conclude that for some choice of $\theta$, we have the following inequality.

$$
\int_{-\infty}^{\infty}|\hat{f}(\theta, r)|^{2} r^{2} d r \leq \frac{1}{2 \pi}
$$

Now the main idea of the proof is that for fixed $\theta$, the function $\hat{f}(\theta, r)$ encodes the integrals of $\mathrm{f}$ over all the planes perpendicular to $\theta$. This idea appears in the theory of the Radon transform. The problem is rotationally invariant, so we may assume that $\theta=(0,0,1)$. Define the averaged function $F(z)$ to be $\int_{\mathbb{R}^{2}} f(x, y, z) d x d y$. Then an elementary calculation shows that $\hat{F}(\xi)=\hat{f}(\theta, \xi)$. Using equation $(*)$, we can estimate $\hat{F}$ sufficiently well to bound $\sup _{z}|F(z)|$.

In addition to $(*)$, we also know that $|\hat{F}(\xi)|=|\hat{f}(\theta, \xi)| \leq 1$ everywhere. We combine these inequalities.

$$
\int_{-\infty}^{\infty}|\hat{F}(\xi)|^{2}(1+|\xi|)^{2} d \xi<5
$$

Next we use the Cauchy-Schwarz inequality to bound $\int_{-\infty}^{\infty}|\hat{F}(\xi)| d \xi$.

$$
\begin{gathered}
\int_{-\infty}^{\infty}|\hat{F}(\xi)| d \xi=\int_{-\infty}^{\infty}[|\hat{F}(\xi)|(1+|\xi|)](1+|\xi|)^{-1} d \xi \leq \\
\leq\left[\int_{-\infty}^{\infty}|\hat{F}(\xi)|^{2}(1+|\xi|)^{2} d \xi\right]^{1 / 2}\left[\int_{-\infty}^{\infty}(1+|\xi|)^{-2}\right]^{1 / 2}<\sqrt{10} .
\end{gathered}
$$

Finally, by the Fourier inversion theorem, we conclude that $\|F\|_{\infty} \leq\|\hat{F}\|_{1}<$ $\sqrt{10}$. In other words, every integral $\int_{\mathbb{R}^{2}} f(x, y, z) d x d y$ is less than $\sqrt{10}$. Since $f$ is the characteristic function of $U$, the intersection of $U$ with each plane $z=$ constant has area less than $\sqrt{10}$.

In the general case $k>n / 2$, the proof is only slightly more complicated. Instead of polar coordinates, one has to average over the Grassman manifold of k-planes in $\mathbb{R}^{n}$, and instead of the Cauchy-Schwarz inequality, one has to use the Holder inequality.

Falconer's theorem does not extend to the case $k=1$ because of the Besicovitch example. I don't know whether it extends to $k$ in the intermediate range $2 \leq k \leq$ $n / 2$. For more information, consult [2], 15], and the references therein.

\section{REFERENCES}

[1] Almgren, F. J., The theory of varifolds - a variational calculus in the large for the kdimensional area integrated, unpublished.

[2] Bourgain, J., Besicovitch type maximal operators and applications to Fourier analysis, Geom. and Funct. Anal. 1 (1991), no. 2, 147-187.

[3] Brooks, R., The spectral geometry of towers of coverings, J. Differential Geom. 23 (1986), no. 1, 97-107.

[4] Burago, D., and Ivanov, S., On asymptotic isoperimetric constant of tori, Geom. and Funct. Anal. 8 (1998) no. 5, 783-787.

[5] Falconer, K. J., Continuity properties of k-plane integrals and Besicovitch sets, Math. Proc. Cambridge Phil. Soc. 87 (1980) no. 2, 221-226.

[6] Fleming, W., Flat chains over a finite coefficient group, Trans. Amer. Math. Soc. 121 (1966), $160-86$.

[7] Gromov, M., Filling Riemannian manifolds, J. Differential Geom. 18 (1983) no. 1, 1-147. 
[8] Gromov, M., Metric Structures on Riemannian and Non-Riemannian Space, Birkhauser, Boston, 1999.

[9] Guth, L., Area-contracting maps between rectangles, PhD thesis, MIT, Department of Mathematics, 2005.

[10] Moser, J., On the volume elements on a manifold, Trans. Amer. Math. Soc. 120 (1965), 286-94.

[11] Nabutovsky, A. and Rotman, R., Volume, diameter, and the minimal mass of a stationary 1-cycle, Geometry and Functional Analysis 14 (2004) no. 4, 748-90.

[12] Pitts, J., Existence and Regularity of Minimal Surfaces on Riemannian Manifolds, Princeton University Press, Princeton, New Jersey, 1981.

[13] Vaaler, J. D., A geometric inequality with applications to linear forms, Pacific J. Math. 83 (1979) 543-553.

[14] Whitney, H., Geometric Integration Theory, volume 21 of Princeton Mathematical Series, Princeton University Press, Princeton, New Jersey, 1957.

[15] Wolff, T., Recent work connected with the Kakeya problem, pages 129-162 in Prospects in Mathematics, (Princeton, NJ, 1996), Amer. Math. Soc., Providence RI 1999.

Department of Mathematics, Stanford, Stanford CA, 94305 USA

E-mail address: lguth@math.stanford.edu 